University of Redlands

\title{
Identifying Suitable Locations to Build External Partnership in Mexico Using GIS Technology
}

A Major Individual Project submitted in partial satisfaction of the requirements

for the degree of Master of Science in Geographic Information Systems

by

Shirley Diana Perez

Fang Ren, Ph.D., Committee Chair

Douglas Flewelling, Ph.D.

December 2011 


\section{Identifying Suitable Locations to Build External Partnership in Mexico Using GIS Technology}

Copyright (C) 2011

by

Shirley Diana Perez 
The report of Shirley Diana Perez is approved.
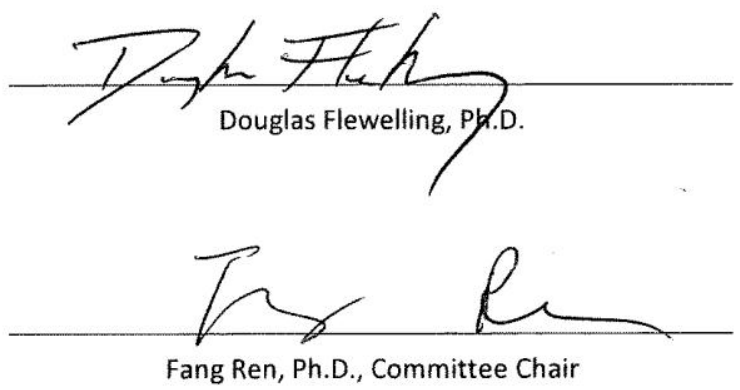

December 2011 



\begin{abstract}
Acknowledgements
I would like to gratefully acknowledge the enthusiastic supervision of Dr. Fang Ren during this work. Her seemingly endless enthusiasm and constant support helped me through the completion of the thesis. I thank the other committee member Dr. Douglas Flewelling for his valuable comments and suggestions. I would also like to thank Dr. Mark Kumler, for his support throughout the program at University of Redlands, and for making the atmosphere in the department stimulating for learning, especially with projections. I am grateful to them and all my friends at the MS GIS program, for being the surrogate family during the year at $\mathrm{U}$ of $\mathrm{R}$ and their continued moral support thereafter. I thank Debbie Riley and Ruben Ortiz for their affection and concern, and for always being there helping each student at any time.

I would like to thank my friends who were always listening to all my complaints, you know who you are, and without doubt you occupy a special part of my life.

I am forever indebted to my family, for their blessings and love and who have supported and encouraged me to do my best in all matters of life. Particular thanks, to my mother, Velia, for her tireless support, love and affection and without whom I would have struggled to find the inspiration and motivation needed to complete this thesis.
\end{abstract}





\begin{abstract}
Identifying Suitable Locations to Build External Partnership in Mexico Using GIS Technology

by

Shirley Diana Perez
\end{abstract}

Building an external partnership is the process in which a firm identifies a portion of its business process that could be performed more efficiently and / or more effectively by another corporation that is specialized in that portion of business. The process of finding external partnerships is long and tedious due to of the large amount of data that must be analyzed. A method was developed using Dr. Johannes Moenius method to reduce the search area of a potential external partnership from a country, to a single city where companies with specific criteria can be found. The model uses GIS technology to more efficiently analyze large volumes of economic data and to integrate them into location analysis to seek for better locations for outsourcing. The project was developed as a desktop prototype application, using Mexico as the study area. This will enable the School of Business at the University of Redlands to use the model in other areas, easily and consistently. 



\section{Table of Contents}

Chapter 1 - Introduction ......................................................................................................... 1

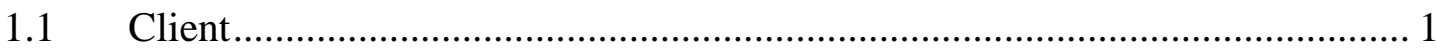

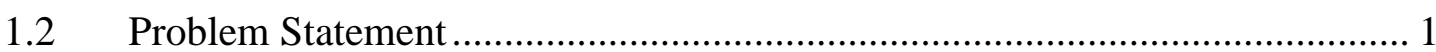

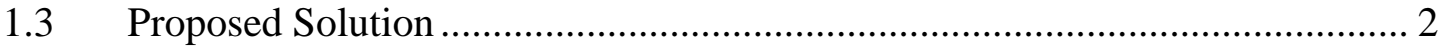

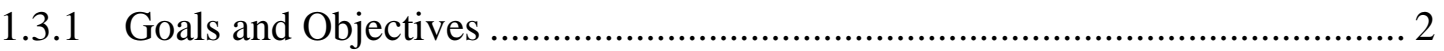

1.3.2 Scope

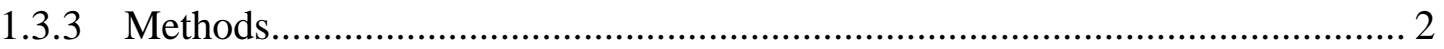

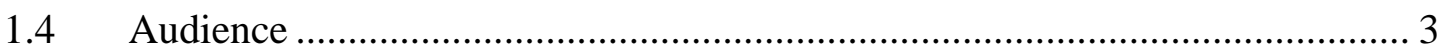

1.5 Overview of the Rest of this Report …………............................................... 3

Chapter 2 - Background and Literature Review ................................................................. 5

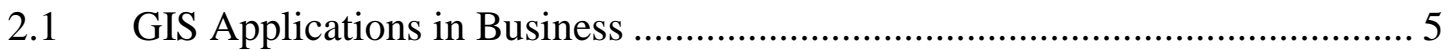

2.2 Location Analysis with GIS for Outsourcing ………….............................. 6

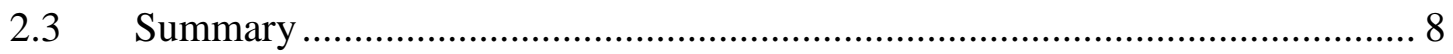

Chapter 3 - Systems Analysis and Design..............................................................9

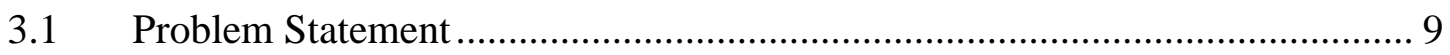

$3.2 \quad$ Requirements Analysis ……………………..................................... 9

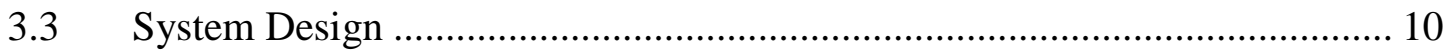

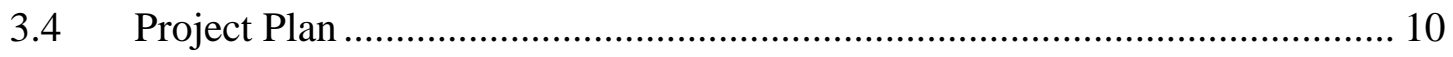

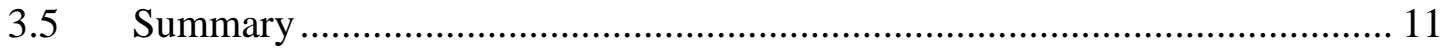

Chapter 4 - Database Design.................................................................................................... 13

4.1 Conceptual Data Model ....................................................................... 13 


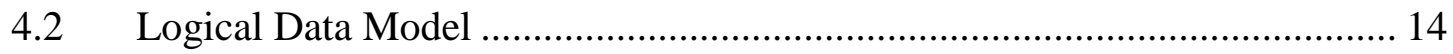

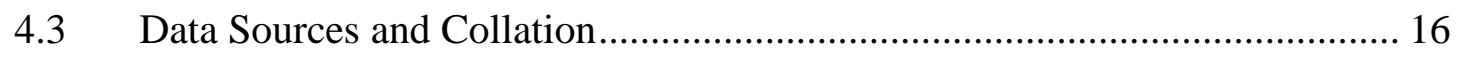

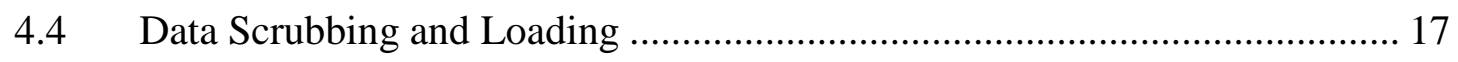

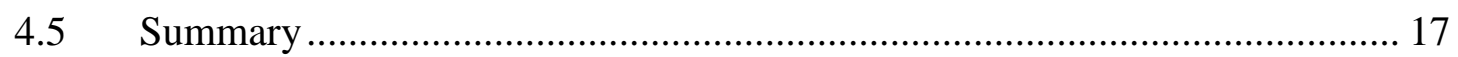

Chapter 5 - Implementation......................................................................... 19

5.1 Economic Variable Standardization .................................................... 19

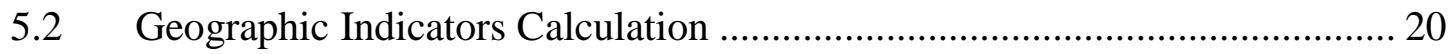

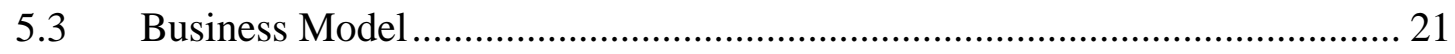

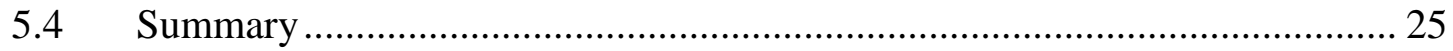

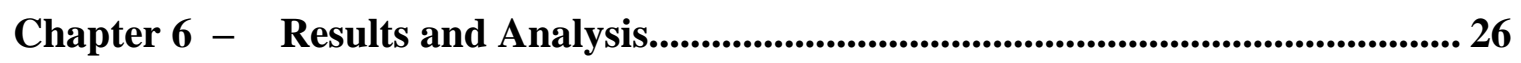

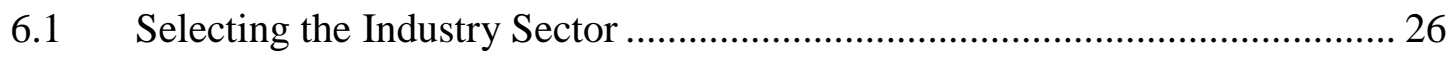

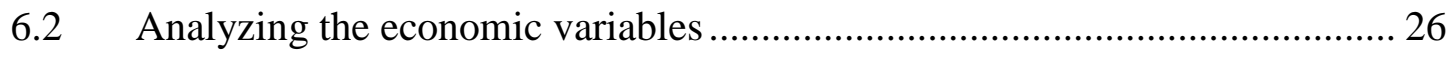

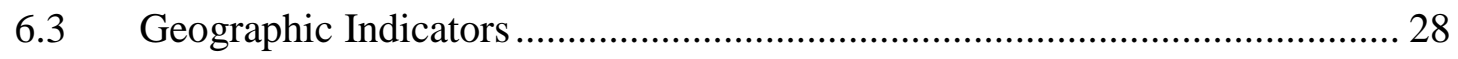

6.4 Production Factors and Geographic Indicators ....................................... 30

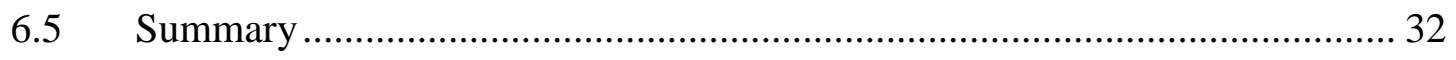

Chapter 7 - Conclusions and Future Work ......................................................... 33

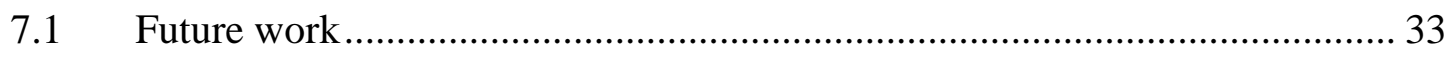

Works Cited $\quad$................................................................... 35

Appendix A. Script for the Production Factors .......................................................... 37

Appendix B. Script for the Local Access ........................................................................... 45

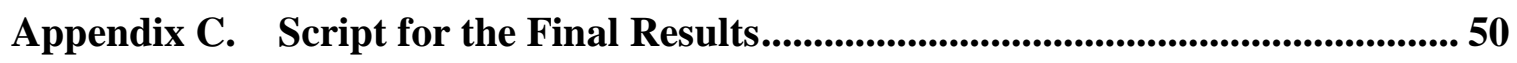




\section{Table of Figures}

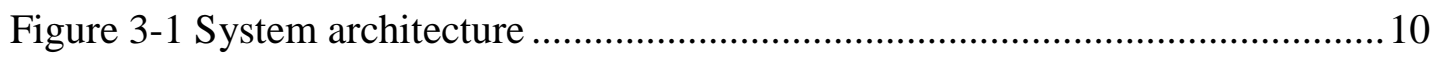

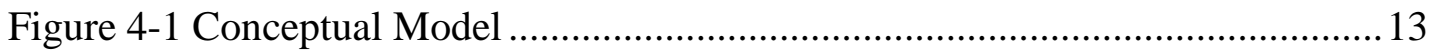

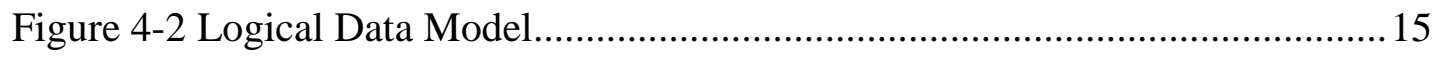

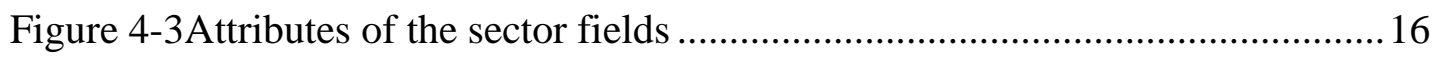

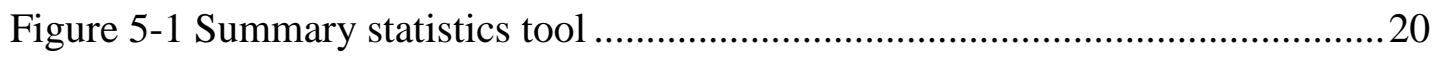

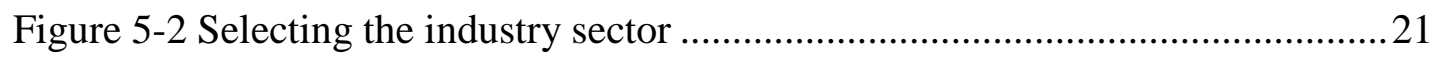

Figure 5-3 Inputting the production factors weights........................................ 22

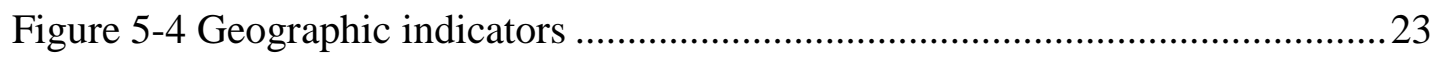

Figure 5-5 Production factors and transportation weights ................................... 24

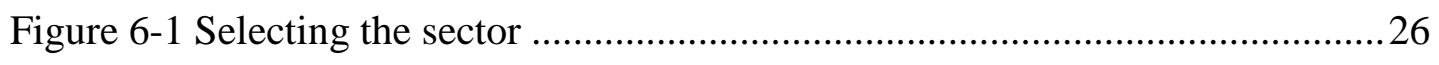

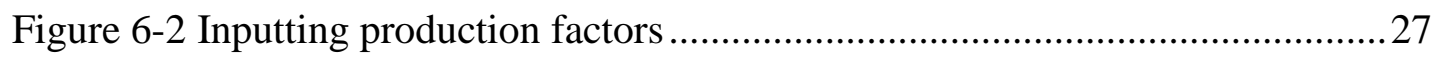

Figure 6-3 Standardized production factors and production factor index ................28

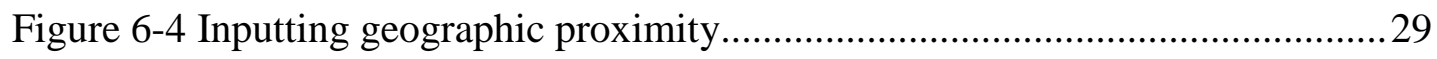

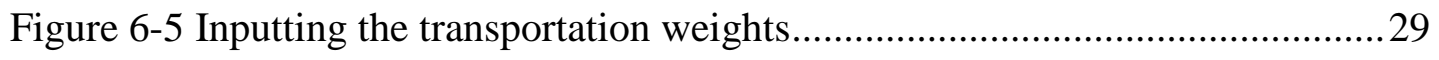

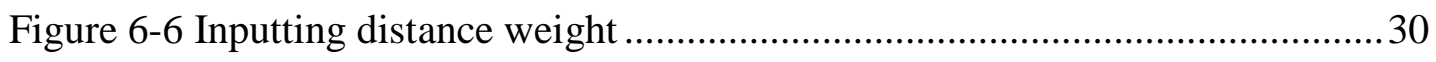

Figure 6-7 Inputting transportation and production factor weights ......................... 30

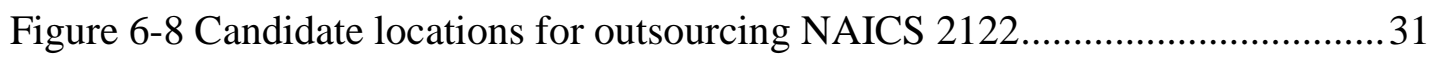

Figure 6-9 Attribute table for the final suitability scores.................................... 32 



\section{List of Tables}

Table 2-1 Production factor variables ..................................................................... 7

Table 3-1Functional and non-functional requirements .............................................. 9

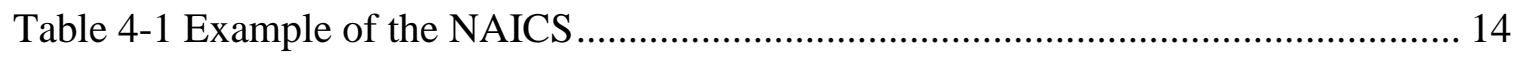





\section{List of Acronyms and Definitions}

GIS- geographic information systems

INEGI- Instituto Nacional de Estadística y Geografía (National Institute of Statistics and Geography)

LA- local accessibility

NAICS- North American Industrial Classification System

PF- production factors 



\section{Chapter 1 - Introduction}

According to an old adage, the three most important considerations in business are "location, location, location." Building an external partnership is the process in which a firm identifies a portion of its business process that could be performed more efficiently and / or more effectively by another corporation that is specialized in that portion of business. This allows the first organization to focus on the central part or function of their business. Before business decision-makers choose a location to build an external partnership and outsource part of their production processes, they need to perform market analysis to find the best place to obtain goods and services. This analysis is time consuming, making the process tedious and long. Thus, the goal of this project was to develop a tool that could facilitate the analysis of searching for a location to collaborate, and then ease the job of the businesses decision makers.

To achieve the project goal, an automated workflow was created using geographic information systems (GIS). With the GIS technology, it was more efficient to analyze large volumes of economic data, and to integrate them into location analysis to seek for better locations for outsourcing. Moreover, since location analysis for outsourcing inherently is a spatial problem, geographic information systems can provide additional insights. For example, distance to the home location of the sourcing firm, proximity to ports, and availability of resources by location, all play important roles in a firm's decisions when trying to outsource to a different location.

\subsection{Client}

The client for this project was Johannes Moenius, Ph.D., the director of the International Business Initiative at the University of Redlands School of Business. Dr. Moenius was looking for a tool that could provide business decision makers with an easier way to locate where goods or services from an outside supplier could be obtained. This is better known as building an external partnership or outsourcing. The client provided the formulations of the business model for this project and determined how to calculate the parameters for the model.

\subsection{Problem Statement}

Analyzing economies in order to locate the best places to outsource has never been an easy task for those in charge of business decisions. On one hand, obtaining a large amount of economic data can be difficult for firms, particularly for small firms that have limited resources. On the other hand, to locate the best locations, a variety of variables in addition to economic variables, such as distance and accessibility, need to be considered. This data analysis becomes complex and is often challenging to conduct. 


\subsection{Proposed Solution}

The proposed solution was to develop a GIS business model that allowed business decision makers to seek new outsourcing locations by accessing readily available and pre-analyzed data. The business model narrowed the number of potential locations where investments can be made within a selected area. In the business model, Mexico served as the place for outsourcing and fictitious U.S. companies were created for the project as examples of potential clients. In the future, data from other countries can be used as well.

\subsubsection{Goals and Objectives}

Finding the best location to establish an external partnership can be one of the most important elements in establishing a lasting and flourishing business. The major goal of this project was to create a business model tool which would reduce the time and costs related to looking for the best places to invest. In doing so, the decision making process will become easier, as the model will consider a variety of factors and present the most beneficial choices for external partnerships. Additionally, the local communities of the target regions could benefit from the establishment of new companies in their area, generating employment opportunities.

\subsubsection{Scope}

The primary goal of this project was to create a tool to facilitate business decision makers to deal with the issues discussed in the project's problem statement. Two major components were included in the project scope: a geodatabase that organizes the processed economic census, and transportation data and a GIS model that automates the formulation of the business model provided by the client.

This project was developed as a desktop prototype application. As a result, the project only included the 2009 economic census data, and the study area was limited to Mexico. It is expected that future revisions and updates will be continuously implemented by the business staff of the School of Business of the University of Redlands. These updates will include ongoing data collection, application improvements, and current cartographic representations of the data.

\subsubsection{Methods}

With the purpose of creating the prototype application, both economic and geographic data for Mexico were collected. After all of the data were processed, the analysis began by determining what variables were to be used and what approach was better suited for the creation of the model. After discussing with the client, both production variables and geographic/transportation variables were included in the model. The model was implemented in ArcGIS using python scripting.

The final model allows the user to select the industry sectors and other specifications. To make the data input convenient, the business model includes specific instructions on what is expected in each of the input fields. With the input requirements, 
the model will run a series of analyses with the economic and geographic data in the database. The best locations will be produced in a map for the user to compare.

\subsection{Audience}

This project has been written for business and GIS professionals intending to implement a better solution for finding international outsourcing sites. Further insight into the development of a low cost alternative solution will interest companies working with limited financial resources.

\subsection{Overview of the Rest of this Report}

This report includes seven chapters. Chapter Two contains a literature review and relevant case studies. Chapter Three discusses the project's design and the requirements analysis. Chapter Four introduces the project's database design, data collection, and data scrubbing and loading. Chapter Five elaborates the implementation to complete the business model in GIS. Chapter Six describes the result and analysis, with a discussion of mistakes made, lessons learned, and suggestions for improvements to the project. Chapter Seven outlines general conclusions and suggestions for future work. 



\section{Chapter 2 - Background and Literature Review}

When a company is looking to expand, establishing an external partnership is a costeffective way to start building foundations in other regions of the same country or even in other countries. Building an external partnership, sometimes also called outsourcing, is the act of one company joining with another company to provide goods and services. Often the tasks that are outsourced can be performed by the company itself, but in some cases there are financial advantages that come from outsourcing. Finding the best location in which to outsource to is important for the company because not only must they save on the cost of the goods and services, they too must consider the cost of transportation. If the locations for outsourcing are not appropriately selected it could be more expensive than if the company had done the task itself.

To locate optimal locations is not easy, as many factors need to be accounted for. For companies, particularly small firms, analyzing a large volume of economic and geographic data may be prohibitively difficult. Therefore, a pre-constructed business model would provide an easy, effective, and more accurate way to find better locations to outsource. For this study, the use of geographic information systems (GIS) technology in the business world was reviewed with the purpose of creating such a business model.

\subsection{GIS Applications in Business}

The adoption of advanced technologies in the business management process is becoming a core competency that every company must have in order to take part in today's global and highly competitive business environment. Several practices for improving business processes have been developed and tested by companies around the world with varying degrees of success. In recent years, for example, the business world has discovered the strength of geographic information systems and the advantages of spatial analysis.

However, due to the lack of exploration, the full potential of GIS technology has not been realized and business communities have been slow to embrace it. Regardless of such hesitation, it has been made clear that the use of GIS by businesses would result in better decision-making, increased efficiency, significant cost benefits, and improved customer satisfaction (Dangermond, 2005).

A geographic information systems is defined as "a computer-based information system that provides tools to manage, analyze, and display attributes and spatial data in an integrated environment" (Mennecke, Crossland, \& Killingsworth, 2000). Because many spatial data analysis functions are incorporated into GIS, it has been widely applied to different business applications. For example, a market analysis can be performed at three spatial levels (Ghosh \& McLafferty, 1987). The first level involves the examination of such factors as population size, demographics, and socioeconomic characteristics like population, income, and existing competition.

The second level of market analysis determines the spatial differentiation of market potential within a selected area. The extents to which demographic and socioeconomic characteristics of the population match the investors' interests are considered. The third level of market analysis is site evaluation. Its objective is to prepare a list of potential sites. 
Another example of use of GIS in business is a project conducted by the SNV Netherlands Development Organization in 2009 (Schill, 2009). The project aimed to generate a lasting impact on improving the lives of poor and disempowered people in Latin America. An inclusive business model was developed as a strategic approach to providing employment and income opportunities for the poor. GIS was intensively used to produce various maps and analyze the different indicators collected through the census. The results of the study provided context information regarding the causes of poverty in the region and facilitated identification of high impact interventions, thus providing ongoing intelligence to support decisions regarding current and new SNV interventions (Schill, 2009).

In addition to analyzing socio-economic data for business applications, GIS has also been used to examine accessibility to different urban opportunities for business planning (Ritsema van Eck \& de Jong, 1999). Traditional location analysis procedures are still employed by many retail companies for site-location. Of the traditional procedures, the most frequently applied techniques for retailers are some form of the analog method, statistical modeling, and gravitational models (Rogers, 2004), which are already implemented in GIS today. Thus, the use of GIS has enabled business decision makers to move away from an intuitive feeling to having factual information relating to a location (Bennison \& Hernandez, 2000). It is no surprise that companies who deploy GIS will have a competitive advantage over companies that do not. For example Ritsema van Eck and deJong (1999) used GIS to study drugstores in the city of Utrecht, Netherlands, performing a spatial analysis of population distribution, the number of people that shopped in the stores and where the shoppers came from. The focus of the study was to find whether more drugstores were required, and to determine the best locations to place them. The site selection analysis in the study was conducted at three different phases.

First, existing facilities were evaluated to estimate their respective market sizes, which may indicate whether some facilities should be relocated or closed. Second, all locations in the area were evaluated for a single new facility to be added to the set of existing facilities; in this way, an accessibility surface can be constructed that can help the decision maker in choosing a suitable site. Third, the decision maker can make one or more proposals for a new location pattern, based either on the existing locations with some relocations and a number of new facilities, or based on completely new locations (Ritsema van Eck \& de Jong, 1999).

This suggests that GIS is a powerful tool for business decision making support; it is more than a mapping tool, which extends much further in its capabilities to analyze spatial information through various spatial analytical tools such as overlays, queries, modeling, statistical comparisons, and optimization (Clarke, 2003).

The growing consumer orientation in business and service planning along with advances in GIS and spatial analysis techniques, have led to the promotion of the use of GIS in the areas of business and service planning (Longley \& Clarke, 1995) by demonstrating the benefits of both methodological advances and evidence of benefits in GIS applications and spatial model in GIS.

\subsection{Location Analysis with GIS for Outsourcing}

Location analysis and GIS are often used in business to solve location related problems, such as determining service areas, finding shortest delivery routes, and selecting an 
optimal site for a new facility to minimize the total travel cost of all potential customers. Besides these business applications, location analysis can also be used for outsourcing purpose. Moenius (2011) came up with an outsourcing business model which considers two broad categories of variables to decide where to find the best resources.

The first category of variables includes all the economic data extracted from the census data that is provided by the government. The model refers to this category of economic variables as production factors. The production factors include fourteen variables calculated from the already existing variables (Table 2-1). The second category is geographic indicators, which includes accessibility and the distant from the company's home location to the locations considered for outsourcing. Accessibility is expressed as the distance from each municipality in Mexico to its closest airport, port, road and railroad.

\section{Table 2-1 Production factor variables}

\begin{tabular}{|l|l|}
\hline No. & Production Variables \\
\hline 1 & Total workers in industry \\
\hline 2 & Remuneration per paid worker \\
\hline 3 & Value added per worker \\
\hline 4 & Gross margin per worker \\
\hline 5 & Share of value added in total production \\
\hline 6 & $\begin{array}{l}\text { Imputed return on capital in percent (= profits }+ \text { interest } \\
\text { avalue added- total remuneration - depreciation)/ fixed } \\
\text { assets } 100\end{array}$ \\
\hline 7 & Capital - labor ratio \\
\hline 8 & Total number of firms in industry \\
\hline 9 & Average size of firm \\
\hline 10 & Labor intensity of value added production \\
\hline 11 & $\begin{array}{l}\text { Depth of production (share of value added in total } \\
\text { production) }\end{array}$ \\
\hline 12 & Revenue per worker \\
\hline 13 & Size of industry (as measured by total revenues) \\
\hline 14 & Asset turnover in years \\
\hline
\end{tabular}

To find the locations where external partnerships could be built, the business model applies three formulas to analyze and calculate the data according to the criteria entered by the user. In this study, the municipalities in Mexico were considered as potential locations. First, the production factor index per municipality (PFI) is calculated as shown in the following formula:

$$
\text { PFI = sum of weighted standardized PF variables }
$$

The PF index summary is the score for each municipality, indicating its suitability for outsourcing based on the economic characteristics of the municipality. The higher score means a better fit for the user's criteria. To calculate the location accessibility index (LAI), the formulas used are as follows:

$$
L A I=\text { sum of [weight } *(1-\text { distance to the mode of transportation/ desired }
$$
distance in miles)] 
The local accessibility index indicates the overall accessibility of each municipality to the four transportation networks (roads, railroads, waterways, and air). The desired distance in the equation is the parameter that will be specified by the user. If a municipality has a greater distance to its nearest airport than what is desired by the user, the computed score for accessibility to the airport will be negative, reducing the final overall accessibility score.

The last formula calculates the final overall suitability score for each municipality, taking in consideration both, the accessibility indicators and the production factors. The formula for the final overall suitability score is:

$$
[(\text { LAI weight } * \text { LAI }+ \text { PFI weight } * P F I) / 100] /[(\text { distance/max-distance } * 100) \wedge
$$

(distance weight /50)]

Distance in the formula is to calculate the distance decay and to eliminate the further distance from the company's home location.

All of these formulas involve weights to evaluate the importance of production factors and geographic indicators in determining the best locations. This is because the role each factor plays may vary across different industries. Therefore, the user is expected to assigns their own weights. Then, according to the company's criteria, the model will calculate the final score of each municipality to decide which one is best.

The aforementioned location analyses can be effectively conducted with GIS technology. On one hand, GIS has the strength to analyze a large amount of socioeconomic data in a spatial context to compare regional characteristics. On the other hand some of the required components in location analysis, such as distance to customers or production locations, spatial accessibility to different kinds of facilities, as well as the availability of resources and competitors nearby, can be conveniently measured and calculated in a GIS. Thus, GIS demonstrates the importance of geographic thinking in business, considers the type of data available and how it can be used, shows how performance indicators can provide management information, and shows how the geographical location analysis processes can aid policy-making (Birkin, Clarke, Clarke, \& Wilson, 1996).

\subsection{Summary}

Geographic information systems have tremendous potential to boost business productivity, and make decisions easier and more effective. Therefore, GIS was applied in this project to create the business model provided by Moenius (2011). The business model can process and analyze large amounts of socio-economic data and transportation indicators, which makes the task of searching for the best locations to build external partnerships easy to fulfill. 


\section{Chapter 3 - Systems Analysis and Design}

This chapter addresses the requirements analysis, system design, and project plan for the development of the business model. Both functional and non-functional requirements of the system were analyzed first before the system was designed. System design addresses the system components as well as how these components were integrated together to create a business model. This was accomplished through developing a customized interface in GIS. Finally, the chapter outlines the original project plan and the modifications that occurred throughout the project.

\subsection{Problem Statement}

Finding the best location to outsource requires considerable market analysis, which is time consuming. By the time the analysis is complete, it could be outdated and of no use to the company. The client needed a tool that allows business decision makers to efficiently find the best location to outsource.

\subsection{Requirements Analysis}

To develop a system that meets client's needs, the requirements of the system should be carefully analyzed. The requirements of a system can be classified into functional requirements and non-functional requirements. Functional requirements describe how the user's needs and productions of useful information are met practically by the system. Table 3-1 summarized the functional and non-functional requirements of the proposed system.

\section{Table 3-1Functional and non-functional requirements}

\begin{tabular}{|c|l|}
\hline Functional/Non-Functional & \multicolumn{1}{|c|}{ System Requirements } \\
\hline Functional & $\begin{array}{l}\text { Business Model to provide the best } \\
\text { locations in the area of interest. }\end{array}$ \\
\cline { 2 - 2 } & $\begin{array}{l}\text { Data of Mexico needed to make the } \\
\text { analysis and find the location. }\end{array}$ \\
\cline { 2 - 2 } & $\begin{array}{l}\text { Data from the user, needed to match their } \\
\text { criteria with Mexico's data. }\end{array}$ \\
\cline { 2 - 2 } & $\begin{array}{l}\text { Interface script to get all the data from the } \\
\text { user }\end{array}$ \\
\hline \multirow{2}{*}{ Non-Functional } & $\begin{array}{l}\text { Use Esri ArcGIS Desktop version } 10.0 \text { for } \\
\text { all processes }\end{array}$ \\
\cline { 2 - 2 } & $\begin{array}{l}\text { Update data when needed to make provide } \\
\text { a more accurate analysis }\end{array}$ \\
\hline
\end{tabular}


The main functional requirement of the system was to develop a business model that would allow the user to identify the best locations that suit the company's criteria. It was assumed that the users may not have previous GIS experience. Because of this, an intuitive interface was required where users could enter their specifications on different parameters, such as the importance of economic and geographic factors. Another functional requirement was to obtain geographic and economic data of Mexico. This is because many of the parameters involved in the business model concerned the economic and market characteristics of different areas in Mexico.

The non-functional requirements were to create the business model that could run in ArcGIS Desktop Version 10.0. This will ensure that the client could keep using the tool in the near future with the minimal modifications. Based on the assumption that the business market is constantly changing, it is necessary for the client to update the database when new datasets are available.

\subsection{System Design}

Based on the requirements analysis, two main components were included in system design: the database and the business model; in addition it was determined that the system would incorporate the user's input data, which could then be used for calculating the best locations to outsource (Figure 3-1). The first step of the design was to create a geodatabase to organize all the variables that are considered by the model for the entire study area. Since the outsourcing analysis requires considering many aspects of the area of interest, the business model should include economic and geographic indicators to evaluate the suitability of an area for a specified industry sector. Because the model itself is rather complicated, an interface was designed to facilitate the user to input all the requirements for the site selection. Both Modelbuilder and python scripting would be required to implement the model and its interface. Once the location analysis is completed by the model, the best candidate locations would be displayed on the map as the final output.

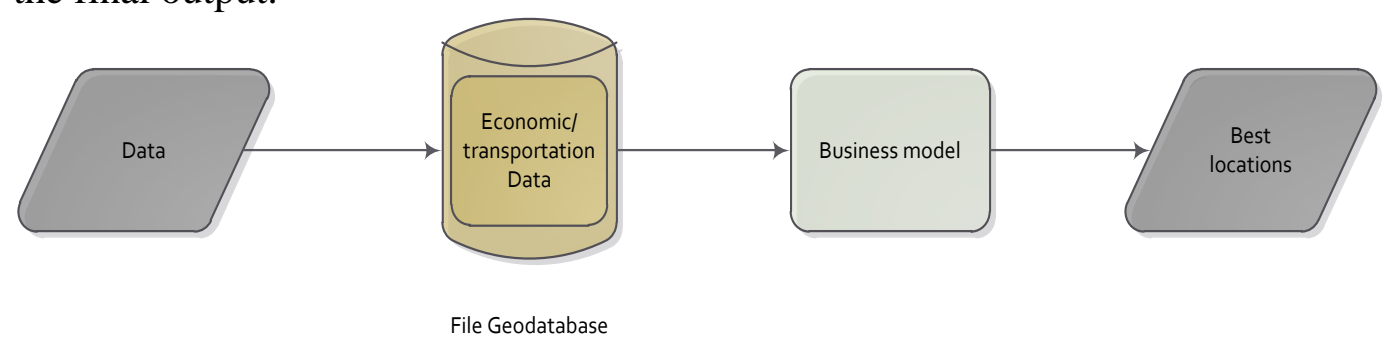

Figure 3-1 System architecture

\subsection{Project Plan}

The project consisted of five major phases. The first phase of the project was to establish the system requirements. This process included meetings with the client and discussing what functional and non-functional requirements to include in order to achieve the desired goal. The following stage focused on literature review, and in this process it was determined to use the model designed by Dr. Moenius to conduct location analysis for outsourcing purpose. 
Data preparation, the third phase, involved organizing and processing the economic and transportation data, this was done to make them ready to be integrated into the model. Several additional attribute fields were created and calculated to produce successful and accurate results. The fourth stage focused on model development, which involved creating the model using Modelbuilder and python scripting. This developed model takes user specified parameter values and runs the analysis to automatically produce the final results. Geodatabase development is the phase that had to be done before the development of the model; crucial to the project was building a file geodatabase that incorporated the economic, transportation, and tools in an organized format, allowing ease of access.

Over all, the implementation of the project went according to the project plan; there were only slight changes made throughout the course of the implementation. Originally the business model was planned to run in a web application, but because of the time given for the completion of the project, the deliverables were changed to a customized model in ArcGIS desktop.

\subsection{Summary}

The completion of the project included the requirements analysis, system design, and project plan for the strategy of the project. The system design was intended to discuss the creation of the Business Model according to the functional and non- functional requirements that were addressed in the requirement analysis. The project plan served to provide a better understanding of the approach that was made and to help prevent the scope from expanding over time. And as with most project plans, this one underwent slight changes that were essential to the completion of the project. 



\section{Chapter 4 - Database Design}

This chapter outlines the database design for the project, which includes: the conceptual data model, the logical data model, and the scrubbing and loading required. The data for the project consists of Mexico economic tabular data that was downloaded from the Internet and transportation data that was created from the professional institutions websites. The methods used to prepare and load the data in the geodatabase are also discussed.

\subsection{Conceptual Data Model}

A conceptual model is a diagram that defines entities, objects, conditions of a system, and the relationships between them. The generalized concept of the project was to find the best locations in which to outsource based on users' preferences. Through a user interface, the user can assign weights to the variables based on their relative importance and the best location for finding external partnerships can be determined accordingly. The conceptual model describes the relationship between the user's company and the goods and services that the company needs. It also describes the relationship of the company with the aggregate unit through the transportation networks (Figure 4-1).

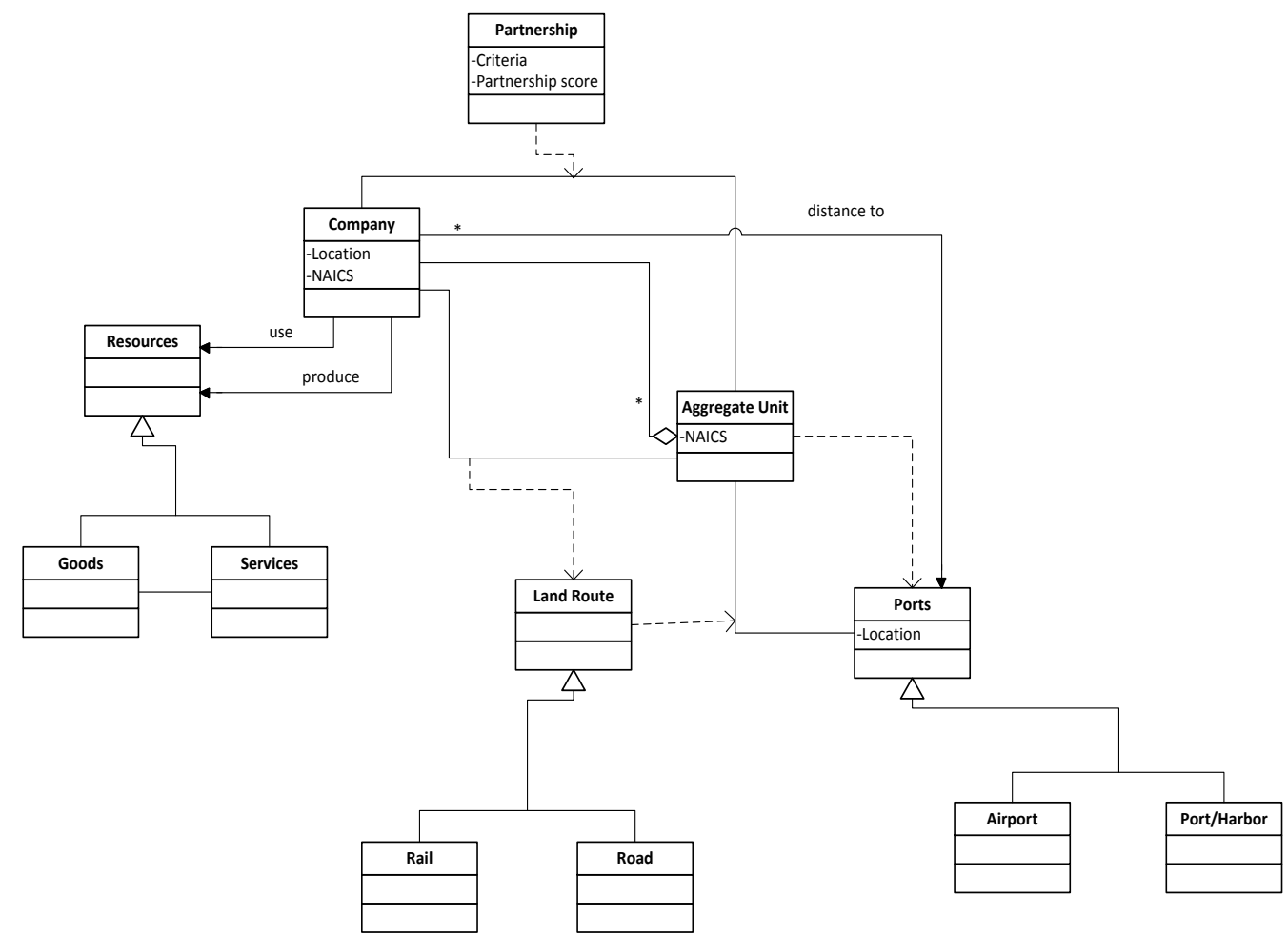

Figure 4-1 Conceptual Model

The Company class provides a unique code to identify the industry specialization that is used by the business model to determine what resources the user is looking for in an external partnership. This code is identifiable, by using the hierarchical coding structure of the North American Industrial Classification System (NAICS). This system is 
used by both industry and government to classify business establishments according to the type of economic activities companies perform. The system employs five-digit codes to represent the hierarchical structure of industry classification. The first two digits designate the largest business sector; the third digit designates the subsector. The fourth digit designates the industry group; and the fifth and final digit designates particular industries. Table 4-1, shows an example of this classification schema.

\section{Table 4-1 Example of the NAICS}

\begin{tabular}{|l|l|l|}
\hline NAICS & Code & Industry \\
\hline Largest business sector & 11 & Agriculture, Forestry, Fishing and Hunting \\
\hline Subsector & 111 & Crop Production \\
\hline Industry Group & 1111 & Oilseed and Grain Farming \\
\hline Particular industries & 11111 & Soybean Farming \\
\hline
\end{tabular}

Another related class to the Company class is the Aggregate Unit class. This class represents the analysis scale, which could be a nation, state or municipality. When a company needs to find a location to build external partnership, they need to decide on which geographic scale their analysis will be based. In this project, the municipality scale was used as the aggregate unit and the analyses were conducted at this level. The Partnership class contains the criteria of the company when looking for the best place to outsource.

Distances to the home location of the company and local transportation accessibility are the concerns when considering where to outsource. In this project four types of transportation networks were considered in the conceptual design: Road, Rail, Air, and Port. The classes Road and Rail belong to the class Land Route transportation, while the Airport and Port/Harbor classes belong to the Port transportation class. The conceptual model also shows the relationship between the Transportation classes and the Company and the Aggregate Unit classes. The relationship between the Company class, the Aggregate Unit, and each of the transportation classes designates the distance from the company's location in the U.S. to each municipality in Mexico. Further, the relationship between the Aggregate Unit class and transportation classes also allows the accessibility of each municipality to different transportation networks to be assessed.

\subsection{Logical Data Model}

The logical data model takes the conceptual model and develops it into the physical geodatabase design phase. A geodatabase was created for organization purposes and data integrity, providing a central location for easy data access and management. The project database was designed in the form of ArcGIS file geodatabase. The geodatabase contains 
multiple feature classes, and the feature classes with similar characteristics are grouped together in a feature dataset within the geodatabase. The geodatabase included three

feature datasets: production factors, transportation data, and aggregate units (Figure 4-2).

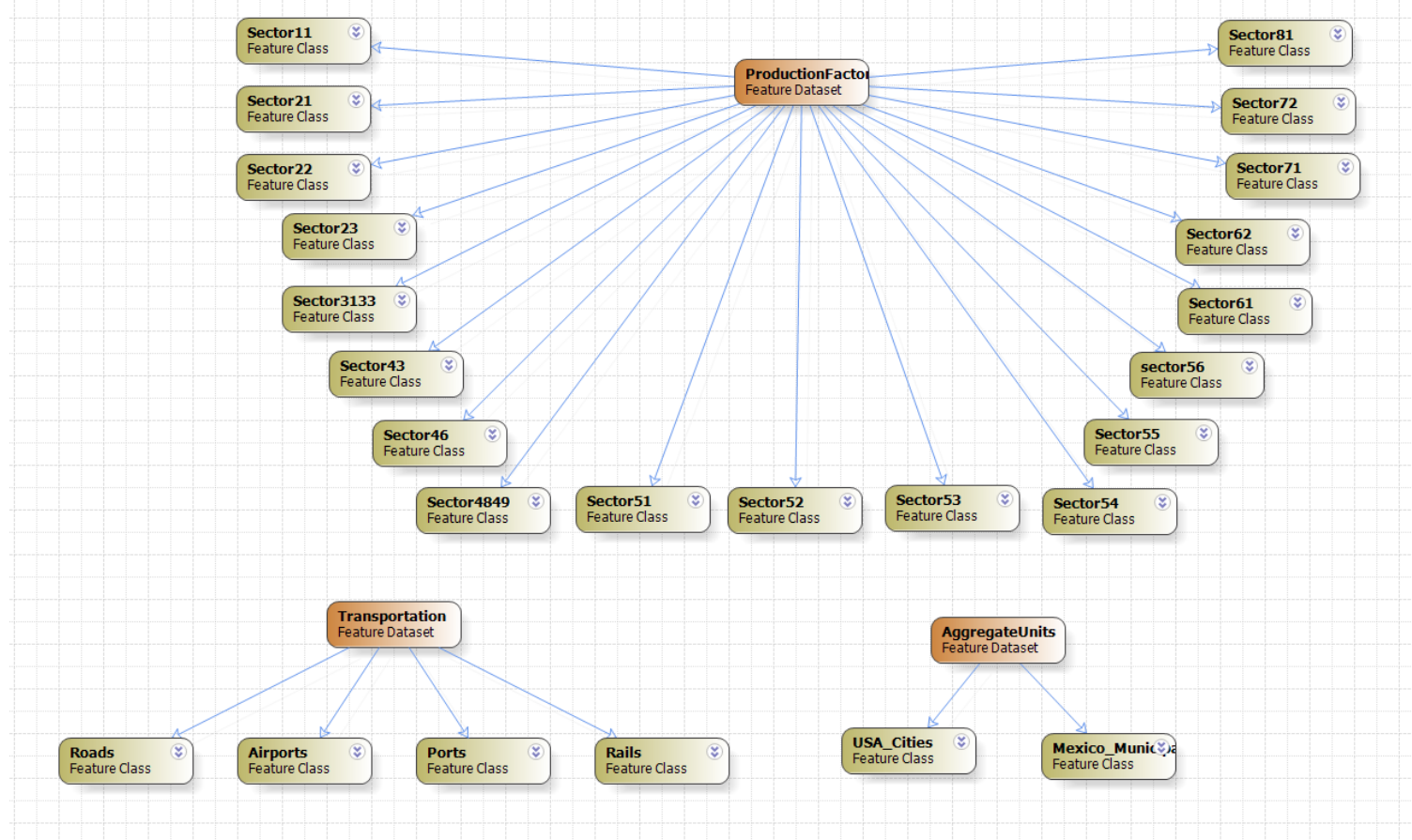

Figure 4-2 Logical Data Model

The production factors dataset contains nineteen feature classes; the name of the feature classes start with "Sector" followed by the first two digits of the sector. Nineteen industry sectors are included in the NAICS: among these, five sectors are mainly goodsproducing sectors, and fourteen are entirely service-producing sectors. The sector feature classes contain the economic data from the 2009 economic census of Mexico. These feature classes contain fourteen economic variables that are used in the business model (Figure 4-3). 


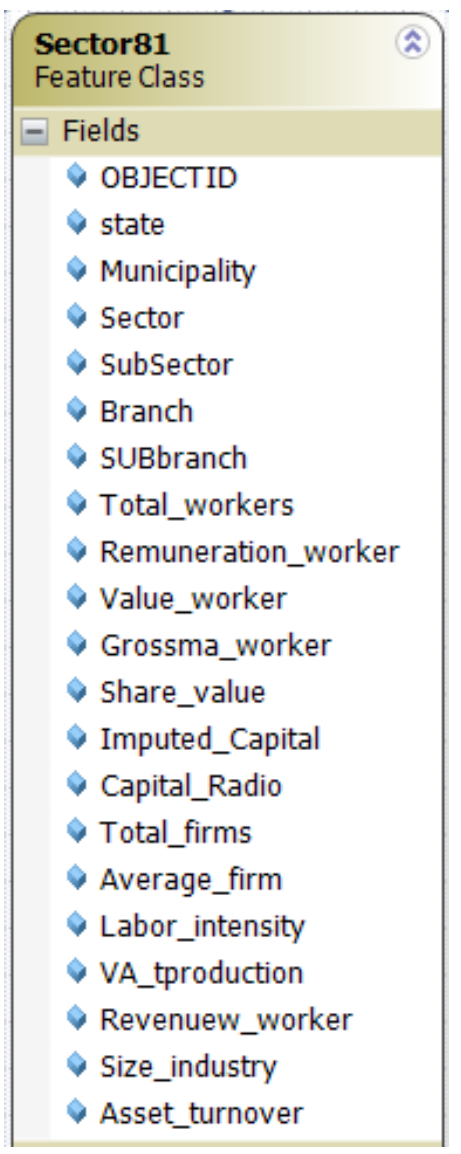

Figure 4-3Attributes of the sector fields

The transportation dataset contains four feature classes which are the four modes of transportations needed for the geographic indicator analysis. The feature classes are: rail, roads, ports, and airports. Each feature class contains the data used to calculate the distance from each municipality to the nearest rail, road, ports, and airports.

The aggregate unit dataset includes two feature classes for the municipalities of Mexico and cities of U.S. The feature class for Mexico municipality contains a set of pre-calculated distances from each municipality to its nearest border entry. These precalculated distances approximate the distance that resources produced in Mexico must travel in order to reach the U.S. For the feature class of U.S. cities, 35,432 cities are considered. This class contains the pre-calculated distance from each U.S. city to the nearest border entry, which approximates the travel distance within the U.S. for goods and services being shipped. In order to make sure that all feature classes shared the same spatial reference, the data was projected to the Azimuthal Equidistant projection.

\subsection{Data Sources and Collation}

Data was collected from three sources for this project. The data used to calculate the economic data for the production factors, was gathered from the 2009 Economic Census from the National Institute of Statistics and Geography (INEGI). The Economic Census was accessed and downloaded in the form of Excel files for every state in Mexico. The 
INEGI website was not user-friendly, and it was difficult to locate the economic data for the 2009 Census. Searching for the correct information in the vast amount of Census data took a great deal of time and effort. Most of the tables only included information at the state level, which was not intended for the desired geographical scale. Further, among the tables that included information at the municipality level, some did not have the right attributes necessary for use. After translating and evaluating more than 10 different types of tables, only one form of these economic tables was found to include the correct information. Fortunately, this type of table was available for all the states in Mexico. A total number of 32 tables of this kind were processed as described in Chapter 5.

All of the data in the U.S. transportation and the Mexico transportation datasets were provided by the Esri data 2010, with the exception of Mexico airports and ports which were created in ArcMap using Excel files. The information for the airports and ports was obtained from the World Port Source webpage.

\subsection{Data Scrubbing and Loading}

In order to prepare the data for the business model, considerable data scrubbing and loading was performed. The economic data needed the most preparation; it required translation from Spanish to English. After the data was translated, it was revised by the client to ensure the variables he wanted used in the business model were included in the tables.

There were 32 tables downloaded; each table corresponded to a state in Mexico. The tables came in Excel format, which contained many variables that were irrelevant to this project. These variables were deleted from the files in order to create a more efficient dataset with only the necessary information. After the tables were cleaned, the client provided the formulas for the calculations to create new variables based on the existing fields in the tables.

Once all of the 32 tables were calculated, all of the tables were put into a file geodatabase, for further processing. This was done because each of these 32 tables only contained all the variables for the municipalities in one state. Since the model would need to evaluate the municipalities across the entire country for one specified industry, it was determined to regroup the tables based on industry. All of the tables were merged together and then divided according to the first two digits of the North American Industrial Classification System (NAICS).

The transportation datasets required minimal data scrubbing and loading as the data provided by Esri came in a format that did not need further preparation. After the addresses for airports and ports in Mexico were put into an Excel file, they were geocoded and the locations of airports and ports were stored in the form of a shapefile.

\subsection{Summary}

Data management is critical for the success of any project. For this project, the conceptual model was the key in the development of a good geodatabase as shown in the logical model. Before the data could be loaded into ArcMap and used for the business model, significant preparations were conducted. The proper preparation of the data and organization of the data in the database provided a solid foundation for the project. 



\section{Chapter 5 - Implementation}

The focus of the project was to develop a business model that could analyze large amount of economic and geographic data and provide the best locations to find external partnerships, taking into consideration the criteria given by the user. Three main components are discussed in this chapter. First, the standardized variable scores needed to be calculated for all fourteen economic variables and for each municipality. With the standardized variable scores calculated, the production factor index could be calculated to indicate the suitability of a municipality for outsourcing in terms of its economic characteristics. Second, local accessibility and the distance from the company's home location to each of the municipalities needed to be computed. Lastly the business model was customized through an interface developed using python in ArcGIS.

\subsection{Production Variable Standardization}

The production factor is an important component that should be considered by the user when deciding the best location for outsourcing. As shown in Chapter 2, the production factor index of each municipality (PFI) is calculated by the sum of each weighted economic variable. However, before applying the formula directly, all the economic variables should be standardized into variable (STDVAR) given the maximum and minimum values of the variables. This is because economic variables are measured at different scales (units).

The equation of standardizing economic variables is as follows:

STDVAR $=[$ value-municipality $-\min ($ municipalities $)] /[$ max (municipalities $)-$ min (municipalities)]

In the equation, the value-municipality is the value of each municipality for each of the economic variables. The min (municipalities) and max (municipalities) is the minimum and maximum value of all municipalities for each of the variables respectively. Therefore a standardized variable of a municipality with a particular industry indicates the relative placement of this municipality for this variable in the industry among all municipalities across the entire country. For example, if the municipality under consideration has the lowest value for a specified variable, such as "total workers" within a specified industry, the standardized score will be zero, indicating this municipality is ranked the lowest in terms of available labor force for this industry in the country.

However, for the variables that have inverse relationship with the suitability for outsourcing, such as the remuneration per paid worker variable, the formula has a slight change:

STDVAR $=\{1-[$ value-municipality $-\min ($ municipalities $)] /[\max ($ municipalities $)-$ min (municipalities)]\}

This is due to the fact that when considering outsourcing, the companies prefer this variable to be lower; it is preferable to negotiate with an external partnership that has low wages for their workers, because that means the resources are going to be cheaper.

This calculation was implemented with ArcGIS ModelBuilder. The minimum and maximum values of each variable were computed using the Summary Statistics tool 
(Figure 5-1). The overall calculation was done by using the Calculate Field tool and it was applied to the economic variables for the industry specified by the user.

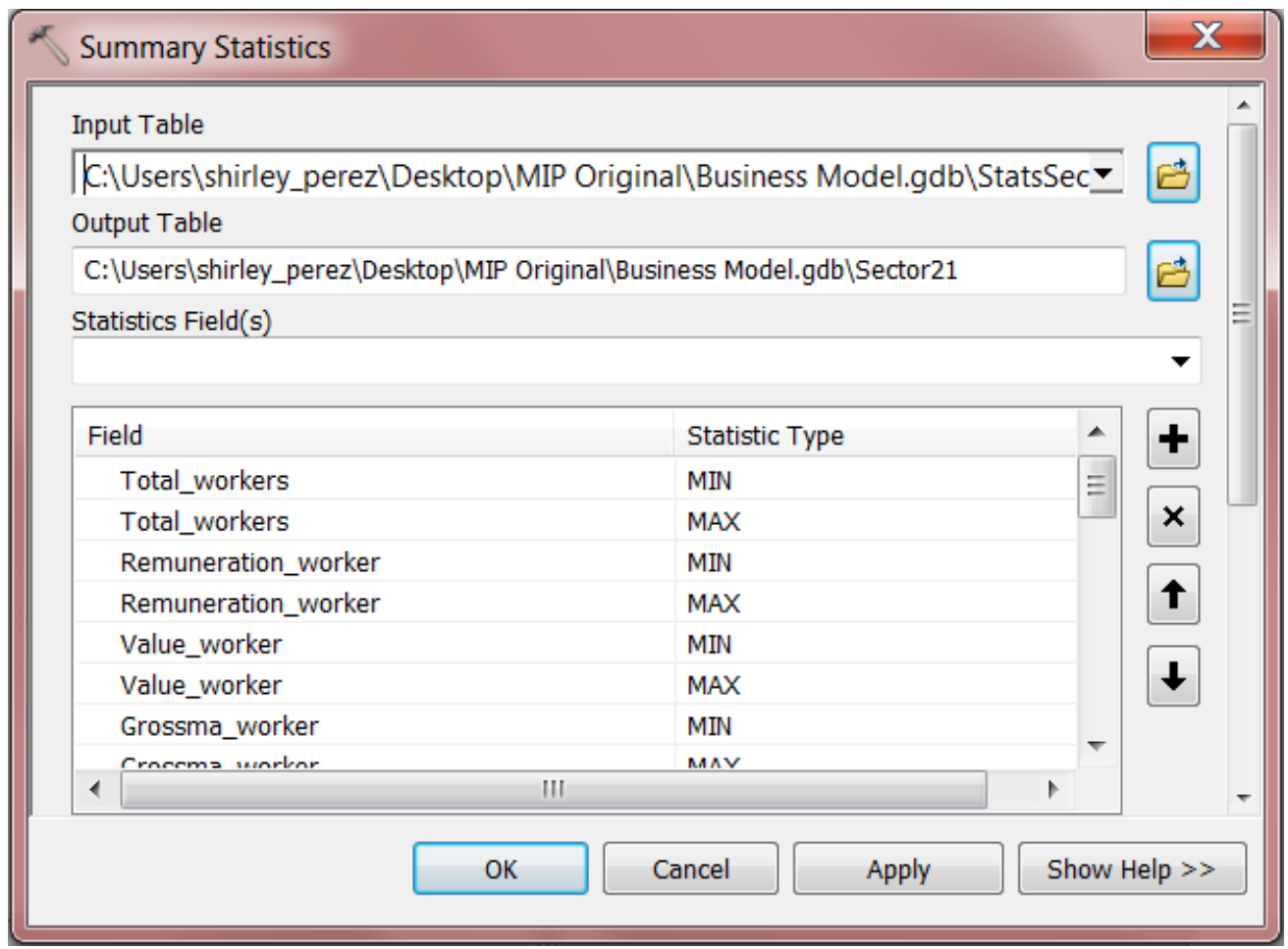

Figure 5-1 Summary statistics tool

\subsection{Geographic Indicators Calculation}

Transportation systems play an important role in shaping the spatial organization of various socioeconomic activities, including retail, manufacturing and services. The role that transportation plays in locating a place for outsourcing was specified by the client of this project. There were two aspects considered for the geographic indicators: the local access and the distance. As discussed in Chapter 2, four kinds of transport, including ports, airports, roads and railroads were considered in the business model. The Near tool was used to pre-calculate the distances from each municipality to its nearest transportation networks, and the distances were used to calculate the local accessibility indices (see details in Section 5.3).

The distance from cities in the U.S. to municipalities in Mexico was the other geographic indicator, which was obtained by calculating the geodesic distance between points. A ModelBuilder was used to calculate the distance between the cities in U.S. and each of the cities in Mexico; this model was used to obtain the most accurate distance. A geodesic distance is the shortest route between two points on the Earth's surface, namely, a segment of a great circle (appendix D). 


\subsection{Business Model}

Standardized economic variables and geographic indicators were formulated together to construct the final business model. The business model was developed by using python script to run in ArcMap and an interface was created for the user to input their specifications.

The interface consists of four parts. The first part is to choose the data to work with, by selecting the geodatabase where all the data are stored and the desired industry sector according to the NAICS code (Figure 5-2).

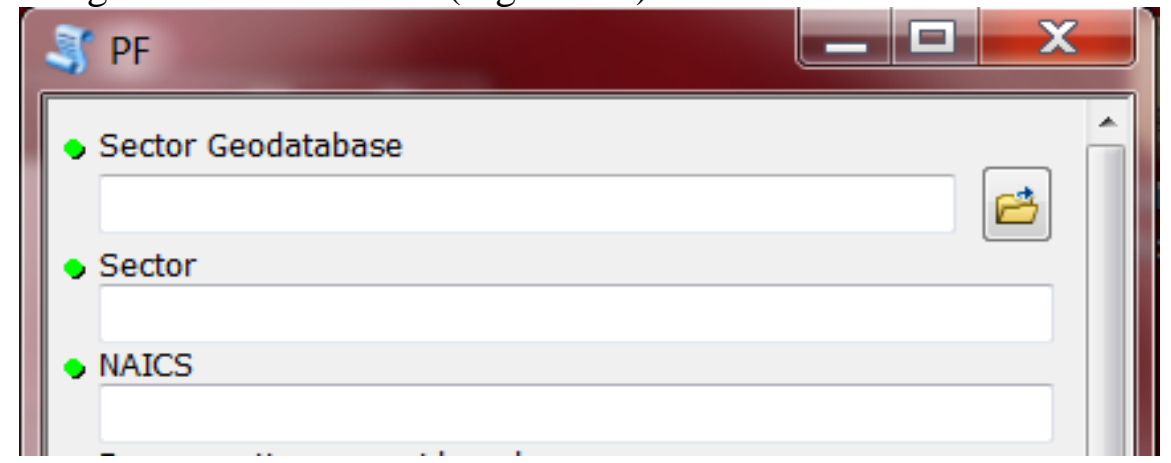

\section{Figure 5-2 Selecting the industry sector}

The second part of the interface is for entering the weights for the economic variables. After the user inputs the weights, the production factor index is calculated by dividing the sum of the productions of each standardized production variable and its corresponding weight by the total weights. This calculation applies to all municipalities. Figure 5-3 shows the interface that the user will use with default weights as zero. The user can drag the slide bar to indicate the relative importance of each factor in determining the preferred location and the corresponding weight will show up in the left textbox. Then the model runs to sum the weighted standardized production factors to calculate the production factor index. 


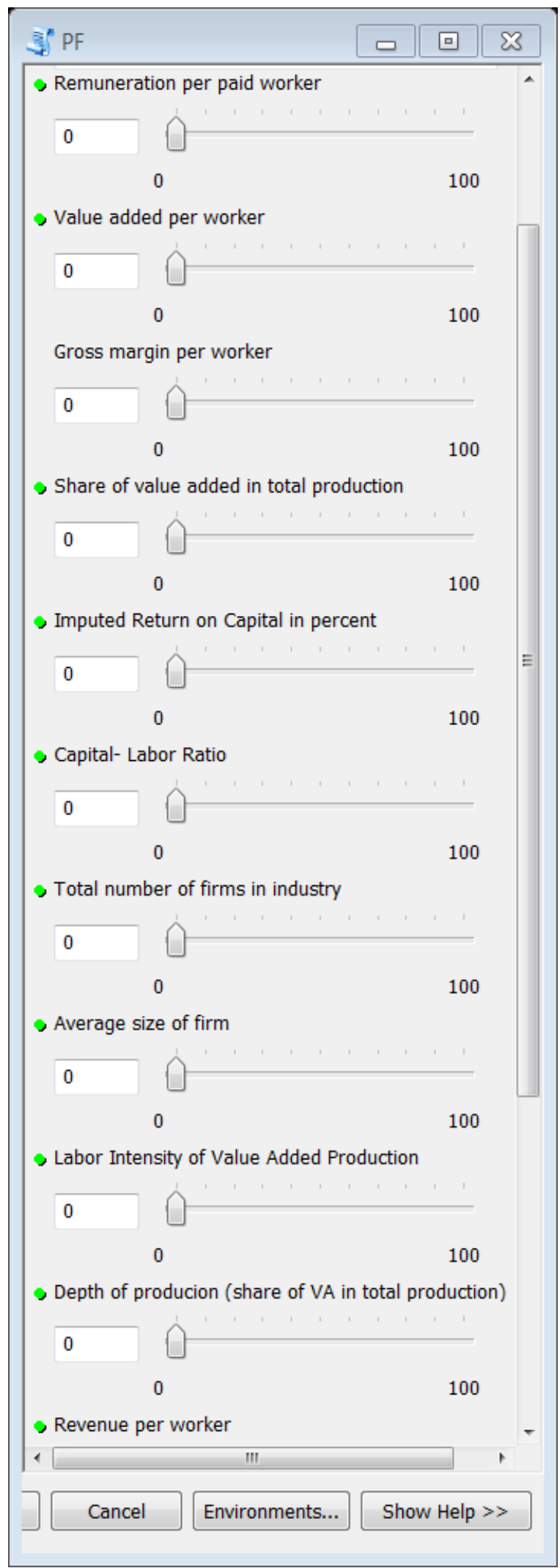

Figure 5-3 Inputting the production factors weights 
The next component is the geographic indicators, which consist of the desired geographic proximity, weights, and, distance (Figure 5-4).

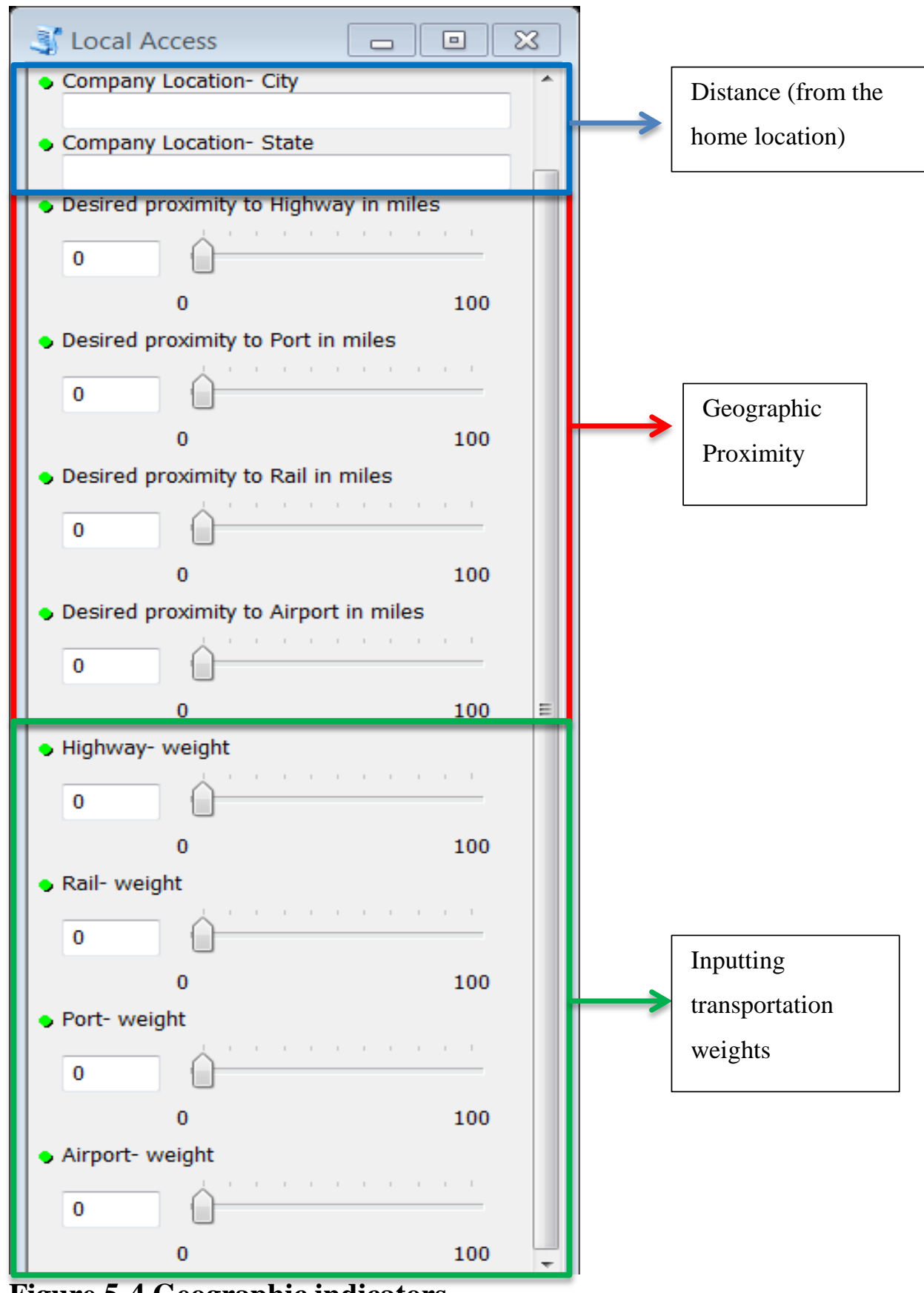

Figure 5-4 Geographic indicators

Once the values have been entered by the user the model calculates the local accessibility (LAI) index using the following formula:

$L A I=$ sum all modes of transportation [user weight $*(1$-actual distance to the mode of transportation/ desired distance in miles)] 
The formula first compares the actual distance to the desired proximity by dividing the actual distance from the municipality to its nearest transportation networks by the desired distances. A large actual distance will lead to a larger proportion, which is less suitable for outsourcing. Therefore, subtracting this proportion from 1 will show the relationship of the actual proximity with the suitability for outsourcing. The local accessibility is then calculated as the sum of weighted proportions.

Based on the home location, distance from the home location to each municipality is calculated by the model. The user's specified weight for distance is then used to calculate the final suitability score as described below.

The last step asks the user to enter the weights for transportation and production factors and the importance of distance (Figure 5-5). The weights between the transportation and production factors determine what aspect is more important to the user.

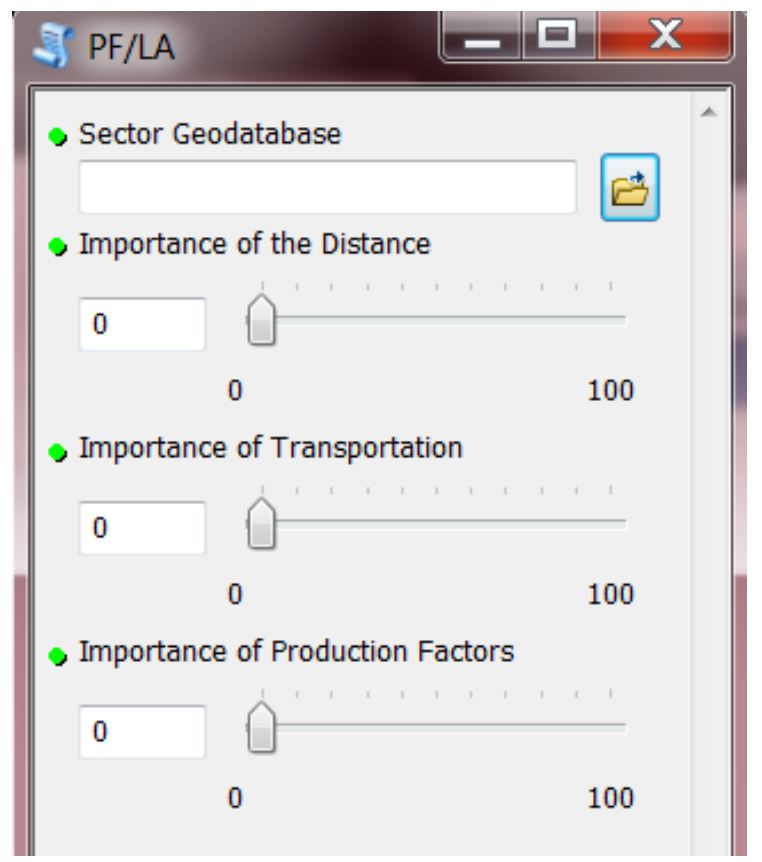

Figure 5-5 Production factors and transportation weights

The business model then finds the best locations that meet the company's criteria based on the following equation:

$$
\begin{aligned}
& \text { Final Score }=[(L A I \text {-weight } * L A I+P F \text {-weight } * P F I) / 100] / \\
& \left\{[\text { distance/max }(\text { distance }) * 100]^{\wedge}(\text { distance-weight } / 50)\right\}
\end{aligned}
$$

Where LAI-weight and PFI-weight are the weights the user gives to the transportation and production factors respectively. The $L A I$ and $P F I$ are the values of each municipality calculated as previously discussed. The distance is the distance from the company's home location to the municipality. The max (distance) is the distance from the location of the user to the furthest municipality. And the distance-weight is the weight value assigned by the user depending on the importance of distance to the company. The client wanted to discard more distant locations according to the preference of the company, because distance exponentially reduces the value of a location. After the 
model finishes processing the information, the suitability of municipalities is calculated and displayed in a map for the user to explore.

\subsection{Summary}

The main focus of the business model is to analyze large amounts of economic data and take into consideration the transportation cost to find the best locations to which a company can outsource. A model was created using python script to run in ArcMap to produce a dialog box that allows the user to enter the company's desired information and criteria. The model then runs and identifies the areas most suitable for the company according to the user specified criteria in the model. 


\section{Chapter 6 - Results and Analysis}

The business model was developed to allow companies to analyze both economic and geographic data to find potential locations for building an external partnership. The model makes the analysis easier by providing an interface where the user has the ability to specify their requirements for outsourcing. This chapter describes a user case where a silver mining company located in Redlands, California, would like to find potential locations for outsourcing in Mexico.

\subsection{Selecting the Industry Sector}

Figure 6-1 shows the first part of the interface that the user will see when launching the model application. Here the user needs to select the geodatabase where the economic and transportation data are stored. The user selects the sector number. Then the user has to select the industry based on the NAICS code.

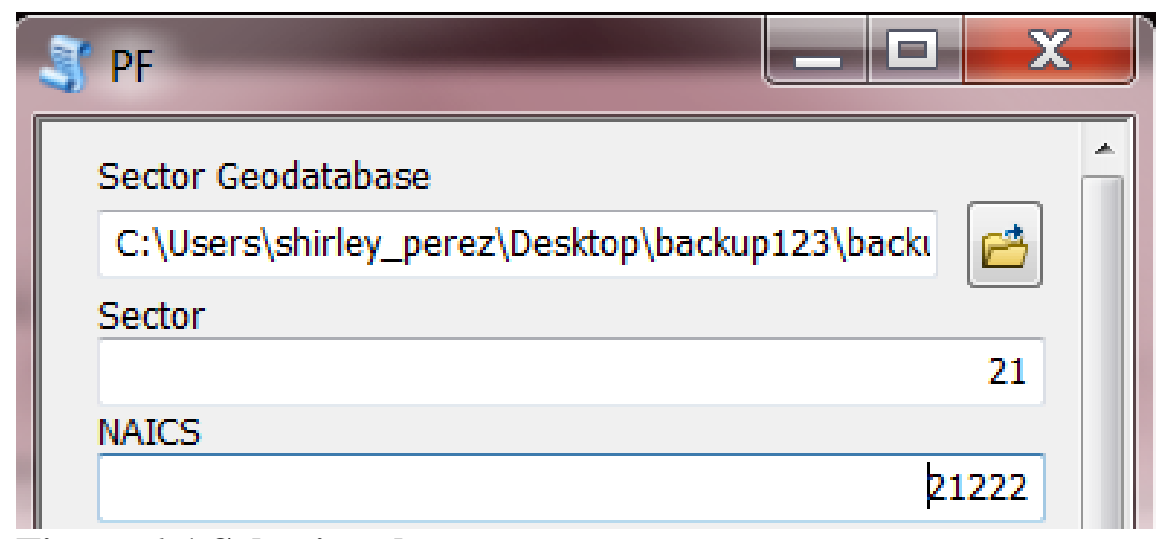

Figure 6-1 Selecting the sector

In this example, the user selected the Sector 21, which belongs to Mining, Quarrying, and Oil and Gas Extraction. The user then entered the NAICS code for the specific industry level of their choice, which was 21222, corresponding to Gold Ore and Silver Ore Mining. The user could have chosen any level of the NAICS code in sector 21, which belongs to Mining, Quarrying, and Oil and Gas Extraction since this was the sector that was selected. The other NAICS that could have been selected following this example, are the subsector, 212, is mining (except for Oil and Gas). The branch 2122 belongs to Metal Ore Mining, and the sub-branch 21222 corresponds to Gold Ore and Silver Ore Mining. Once this selection is made, the model will only work with the municipalities that have Industry NAICS code 21222.

\subsection{Analyzing the economic variables}

In this part of the model, the production factor index is calculated based on the company's criteria of the fourteen economic variables. The user is asked to enter the 
weights for each of the economic variables according to the importance of each variable in the specified industry (Figure 6-2).

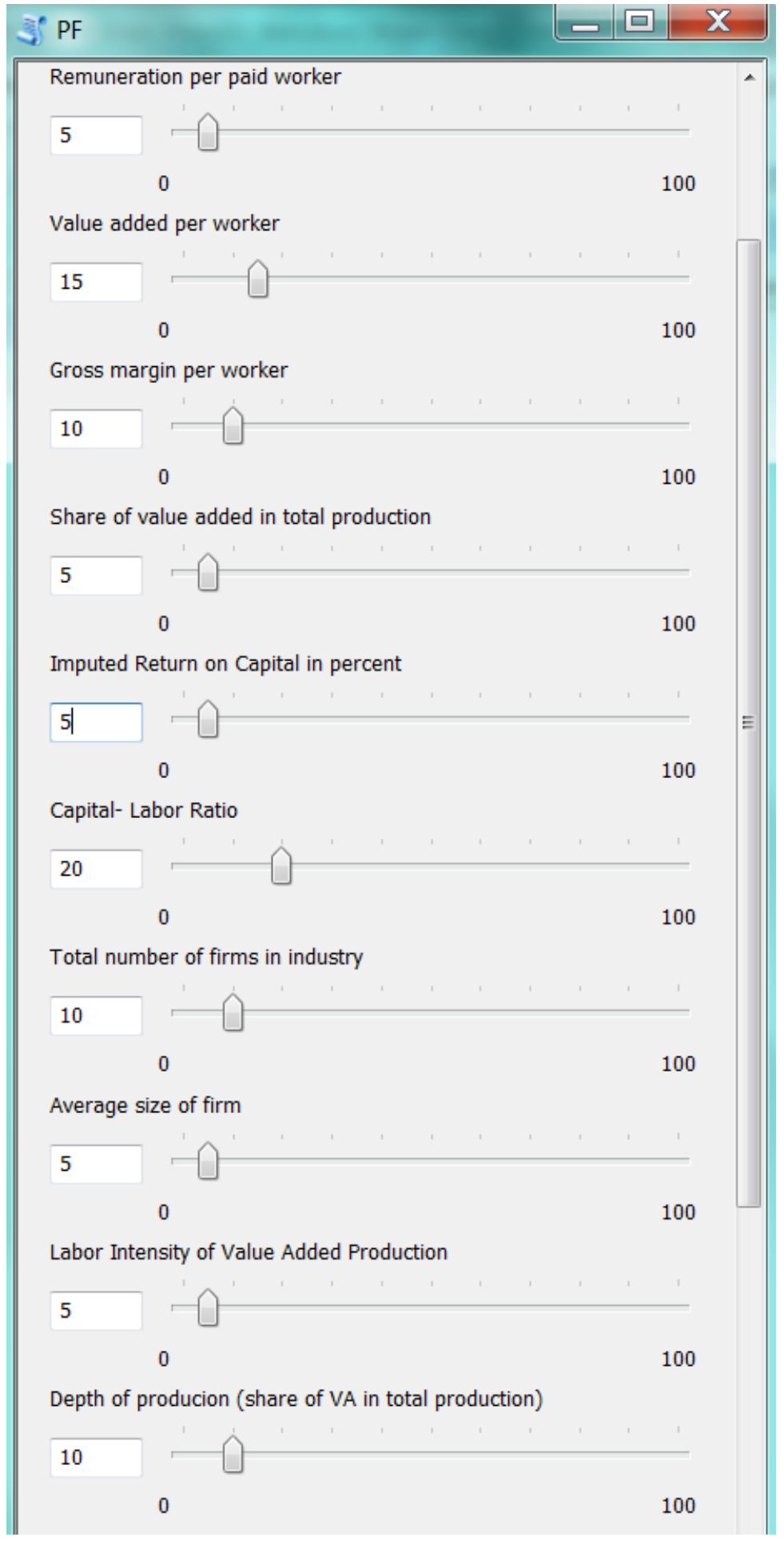

Figure 6-2 Inputting production factors 
In this example, the most important production aspect is the "Capital- Labor ratio", which means that the municipalities that have higher capital- labor ratio value are more desirable. After the weights are entered, the model creates fourteen new fields in the attribute table of the feature class (in this case it is Sector21) to store the standardized economic variables. The weighted fourteen new variables will then be added to obtain the production factor index of the silver mining industry for every municipality in each industry. Figure 6-3, shows six of the standardized economic variables and the last field "PF_Index" containing the PF index. The higher value corresponds to the better suitability for outsourcing when only the economic factors are considered.

\begin{tabular}{|r|r|r|r|r|r|r|}
\hline STD_Labor & STD_VA & STD_Revenue & STD_Sizelnd & STD_AssetT & STD_TWorkers & PF_Index \\
\hline 0.038677 & 0.049883 & 0.001561 & 0.000037 & 0.000003 & 0.000501 & 0.277844 \\
\hline 0.041329 & 0.049354 & 0.000477 & 0.000038 & 0.000005 & 0.001662 & 0.276843 \\
\hline 0.038677 & 0.049684 & 0.001562 & 0.000047 & 0.000003 & 0.000632 & 0.276275 \\
\hline 0.038904 & 0.049761 & 0.00132 & 0.000086 & 0.000002 & 0.001363 & 0.274076 \\
\hline 0.038677 & 0.049543 & 0.000925 & 0.000021 & 0.000001 & 0.000478 & 0.270508 \\
\hline 0.038708 & 0.049533 & 0.000673 & 0.000055 & 0.000002 & 0.001708 & 0.269692 \\
\hline 0.038948 & 0.049837 & 0.000822 & 0.000043 & 0.000002 & 0.001092 & 0.267765 \\
\hline 0.039242 & 0.049404 & 0.00064 & 0.000033 & 0.000007 & 0.001095 & 0.266985 \\
\hline 0.046857 & 0.04927 & 0.000056 & 0 & 0.000006 & 0.00005 & 0.266662 \\
\hline 0.039034 & 0.049699 & 0.00027 & 0.000008 & 0.000001 & 0.00062 & 0.265938 \\
\hline 0.038796 & 0.049545 & 0.001241 & 0.000032 & 0.000003 & 0.00054 & 0.265399 \\
\hline 0.039232 & 0.049802 & 0.00025 & 0.000031 & 0.000002 & 0.002625 & 0.265179 \\
\hline 0.038955 & 0.049818 & 0.000317 & 0.000001 & 0.000735 & 0.000041 & 0.264603 \\
\hline 0.038677 & 0.049644 & 0.000879 & 0.000007 & 0.000002 & 0.000175 & 0.264306 \\
\hline 0.04091 & 0.049343 & 0.000197 & 0.000004 & 0.000001 & 0.000431 & 0.264281 \\
\hline 0.039588 & 0.049621 & 0.000479 & 0.000009 & 0.000001 & 0.000395 & 0.263263 \\
\hline 0.039381 & 0.049588 & 0.000332 & 0.000017 & 0.000002 & 0.001074 & 0.263049 \\
\hline 0.040287 & 0.049534 & 0.000175 & 0.000002 & 0.00001 & 0.000286 & 0.262422 \\
\hline 0.039333 & 0.049452 & 0.000459 & 0.000009 & 0.000003 & 0.000401 & 0.262321 \\
\hline
\end{tabular}

Figure 6-3 Standardized production factors and production factor index

\subsection{Geographic Indicators}

In addition to economic variables, the geographic indicators of a location also have an important role to play. The municipalities that are closer to the home location of the company and have better accessibility to different transportation networks are more suitable for outsourcing as transport cost will be less.

The geographic indicators part of the interface requests the user to input three kinds of criteria: the desired geographic proximity, the weights for each of the modes of transportation, the weight for distance between company's home location and the potential location. Figure 6-4 shows the desired geographic proximity between the potential location for outsourcing and its nearest highway, railway, port and airport. 


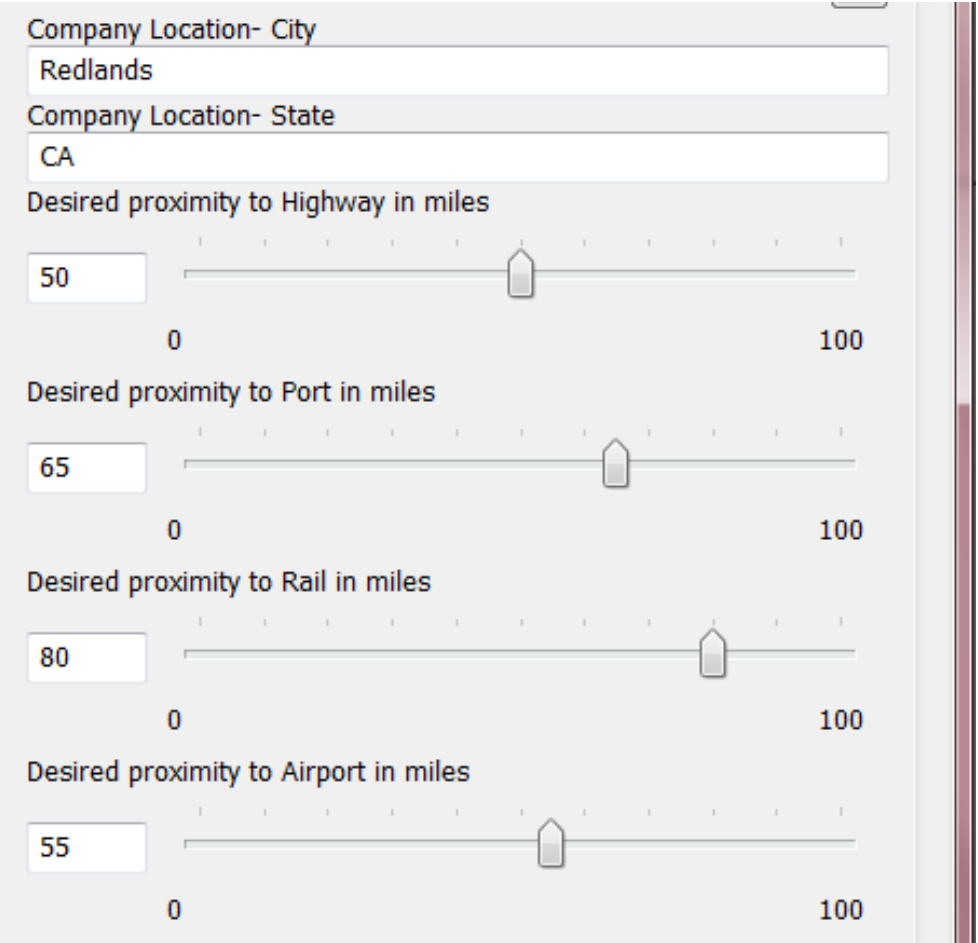

\section{Figure 6-4 Inputting geographic proximity}

The weights for each mode of transportation are specified as well (Figure 6-5). In this example, highway transport is the most important transportation mode for the company and therefore it is assigned the highest weight.

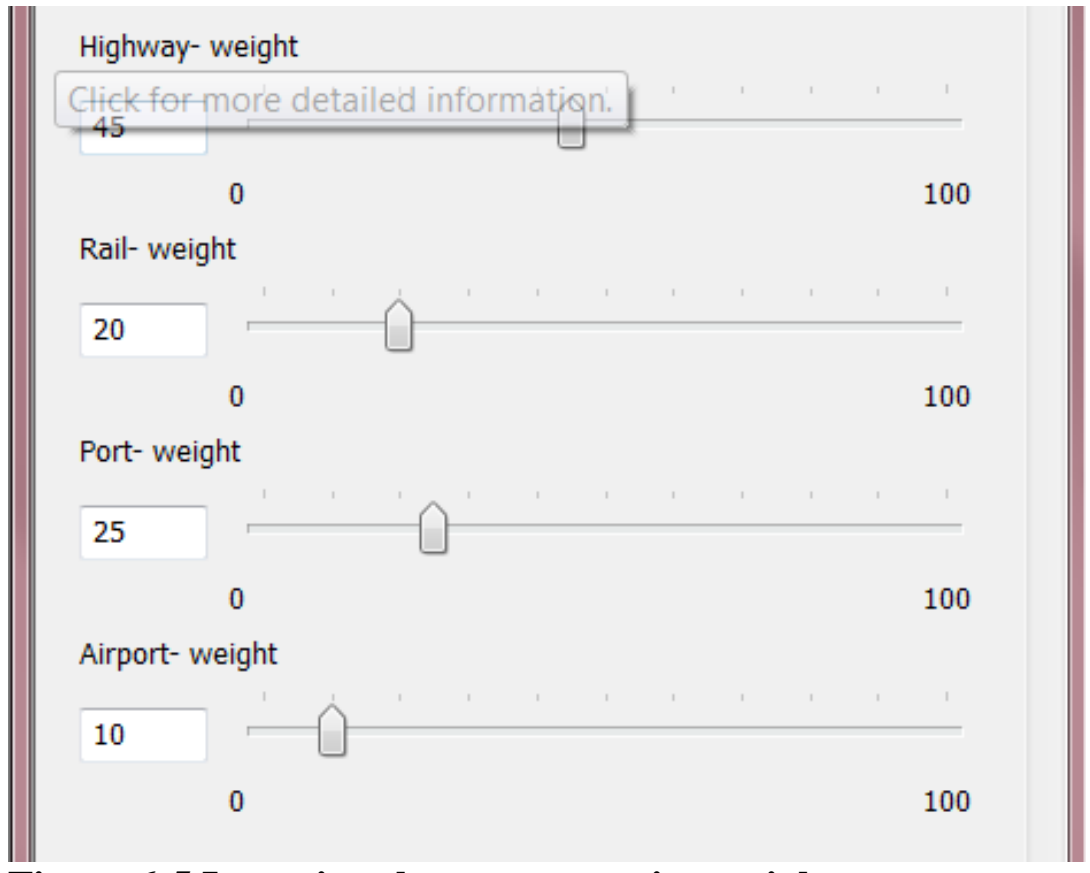

Figure 6-5 Inputting the transportation weights 
After the geographic proximity and the transportation weights have been entered, the model puts them into the calculation to obtain the local access (LA) index. Similar to the $\mathrm{PF}$ index, the greater value of the LA index indicates the higher suitability for outsourcing.

Another component of the geographic indicators is how important the distance is for evaluating the suitability of a location (Figure 6-6). The range of the parameter is from 0 , which means that the distance does not matter, to 100, indicating distance is extremely important. In this example, the importance of the distance is moderate for the company.

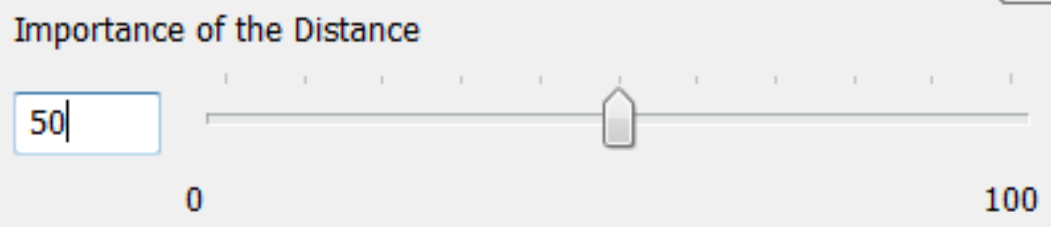

0

\section{Figure 6-6 Inputting distance weight}

Using the home location of the company, the model will first estimate the distance between Redlands, CA. to each municipality. The actual distance and the maximum distance are used to compute the distance decay effect for each municipality (see Chapter 5). The potential interaction between the company and each municipality exponentially decreases with the increase in the actual distance.

\subsection{Production Factors and Geographic Indicators}

The last part of the business model, asks the user to input the weights for the production factor index and local accessibility index (Figure 6-7). This part of the model takes in consideration relative importance of economic variables, the geographic indicators, and the distance of the home location of the company to the municipalities.

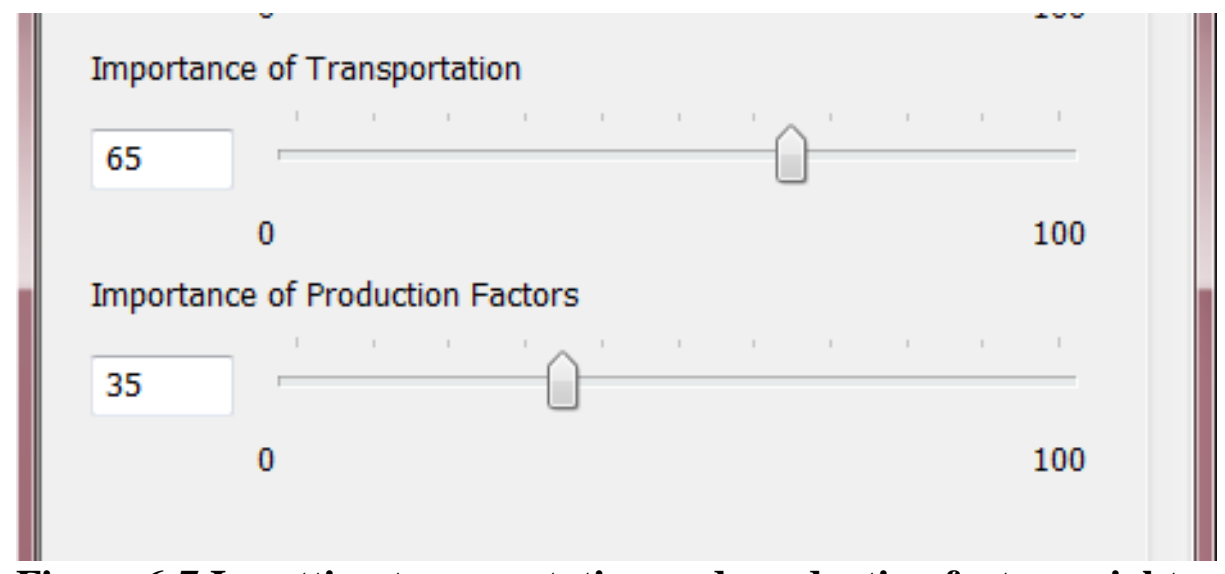

Figure 6-7 Inputting transportation and production factor weights

The example in Figure 6-7 indicates that local accessibility is more important than the production factor in evaluating the suitability of a location for outsourcing. Once all 
the required information is specified, the model runs to produce the final results (Figure 6-8).

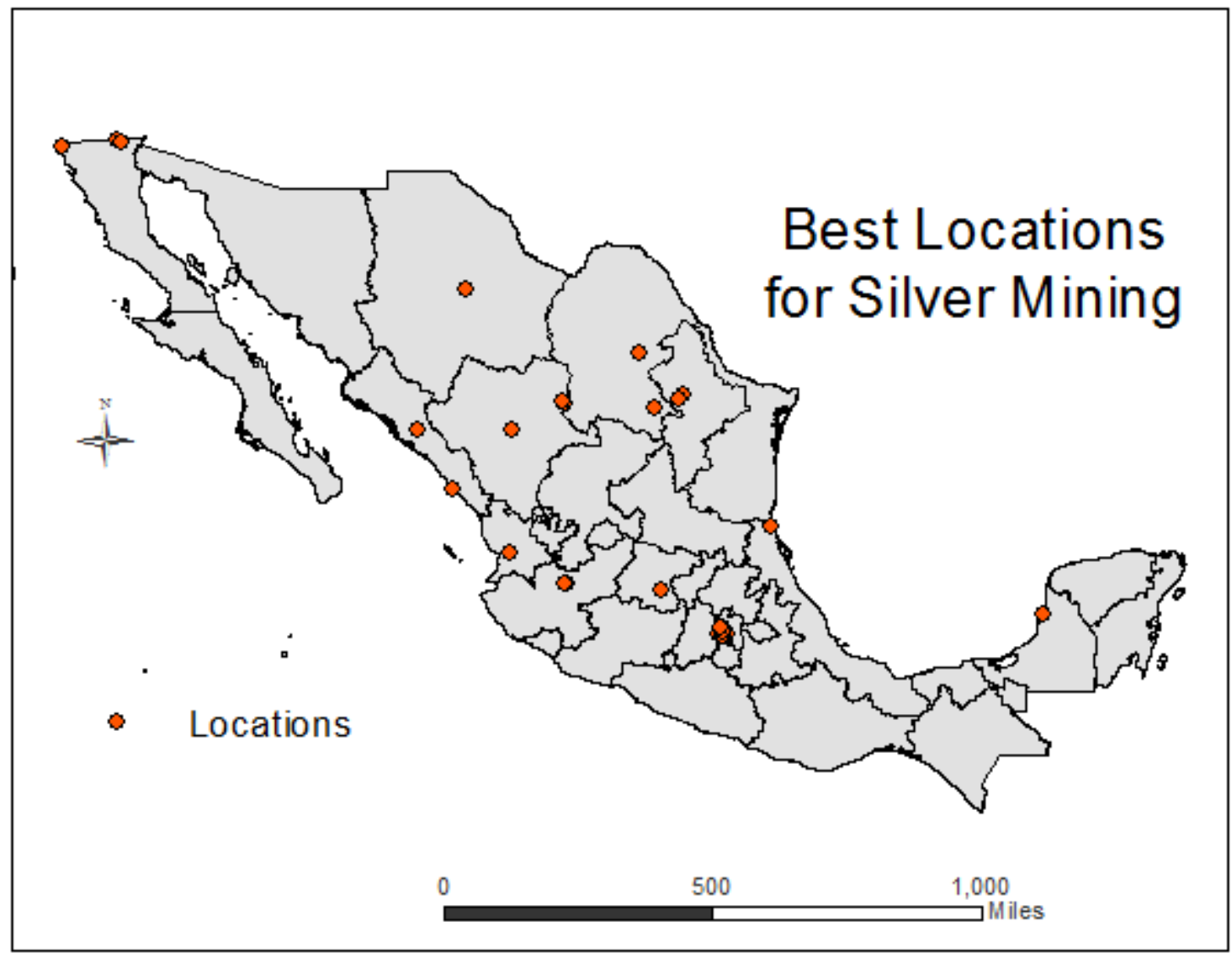

Figure 6-8 Candidate locations for outsourcing NAICS 2122

Among the candidate locations for the external partnership, the user now has the option to choose the location from those recommended by the model by looking at the final suitability scores in the attribute table (Figure 6-9). 


\begin{tabular}{|r|r|r|}
\hline \multicolumn{1}{|c|}{ Final_transportation } & total_distance & Final_suitability_scores \\
\hline 42.24832 & 438.407205 & 0.522809 \\
\hline 56.538132 & 372.307653 & 0.824741 \\
\hline 12.447995 & 361.552086 & 0.188862 \\
\hline 13.252075 & 383.958087 & 0.188797 \\
\hline 44.111703 & 538.795838 & 0.444107 \\
\hline 46.178334 & 557.567713 & 0.450031 \\
\hline 24.454022 & 384.216254 & 0.346665 \\
\hline 67.425502 & 482.514796 & 0.75866 \\
\hline 24.454022 & 384.216254 & 0.346665 \\
\hline 24.454022 & 384.216254 & 0.346665 \\
\hline 45.592413 & 339.450303 & 0.728554 \\
\hline 32.981987 & 278.604633 & 0.644263 \\
\hline 47.884533 & 42.028556 & 6.192382 \\
\hline-19.990965 & 143.357957 & -0.75022 \\
\hline 9.132753 & 62.297345 & 0.805294 \\
\hline 24.454022 & 384.216254 & 0.346665 \\
\hline 24.454022 & 384.216254 & 0.346665 \\
\hline
\end{tabular}

Figure 6-9 Attribute table for the final suitability scores

\subsection{Summary}

The goal of the project was to build a model that could provide an easier way to analyze large amounts of data in order to find the best locations to outsource. Economic data had to be analyzed to find the best resources and transportation costs in order to get the most out of outsourcing. The project goal was accomplished as the user can input their criteria in the dialog boxes to produce the best locations for potential external partnerships. A successful script was created that allows the user to input their own criteria and preferences as well specify distance from the company's home location. In summary, the model produces accurate analysis, making this a successful project. 


\section{Chapter 7 - Conclusions and Future Work}

The most prominent advantages of outsourcing seem to be the fact that it often saves the company money, and it provides more time for the company to focus on more important parts of their business. If a company is looking to expand, outsourcing is a cost-effective way to start building foundations in other states or countries, especially when the company is a small firm. However, the process of outsourcing can be difficult because the business's decision makers have to analyze large amounts of economic data and make spatial analyses such as transportation costs. The client wanted to facilitate the job of business decision-makers in finding a location where they could build external partnerships. To address the problem, a tool was created to run in ArcMap to provide possible locations where external partnerships could be found that met the company's criteria. Some formulas developed by the client were used, along with economic data from Mexico. Geographic indicators were also considered in the model to provide a spatial analysis and to maximize the profits from outsourcing.

Once the calculations were made, the model displayed the results in a map showing the locations where potential companies could get their goods and services from external partnerships. All functional and non-functional requirements laid out by the client were met by the business model. Most importantly, the model can be used by users who have little to no GIS knowledge.

\subsection{Future work}

Although all of the requirements were met, there are additional areas which allow for continuations of this project. The most valuable future work for this project would be to create a web interface for the business model. This would allow the project to reach a wider audience, and make it easier for companies who do not have access to ArcGIS software. The focus of this project was to develop a model using Mexico's data, but the model could be used with any datasets from other study areas.

A third possible area of future work would be to create a model that would not only outsource to Mexico, but could also be used to find the best locations to export to Mexico. That way the tool could be more beneficial to companies. 



\section{Works Cited}

Rodrigue, J.-P., Comtois, C., \& Slack, B. (2009). The Geography of Transport Systems. New York: Routledge.

Bennison, D., \& Hernandez, T. (2000). The art and science of retail location decisions. International Journal of Retail \& Distribution Management, 28(8), 357-367.

Birkin, M., Clarke, G., Clarke, M. P., \& Wilson, A. (1996). Intelligent GIS: Location Decision and Strategic Planning. Wiley.

Boyles, D. (2002). GIS means business. Redlands: ESRI Press.

Clarke, K. (2003). Getting started with geographic information systems. Upper Saddle River: Prentice Hall.

Dangermond, J. (2005). Foreword. In J. B. Pick, Geographic Information Systems in Business.

de Jong, T., \& Ritsema van Eck, J. (1997). Threshold surface as an alternative for location service centres with GIS. Proceedings of the Joint European Conference on Geographical Information (pp. 799-808). Amsterdam: IOS Press.

Ghosh, A., \& McLafferty, S. L. (1987). Local Strategies for Retail and Services Firms. Lexington Books.

Grimshaw, D. (2000). Bringing geographical information systems into business. New York: John Wiley \& Sons.

Longley, P., \& Clarke, G. (1995). GIS for business and service planning. Cambridge.

Mennecke, B. E., Crossland, M. D., \& Killingsworth, B. L. (2000). Is a map more than a picture? The role of SDSS technology, subject characteristics, and problem complexity on map reading and problem solving. MIS Quarterly, 601-629. 
Mitchell, A. (1999). The ESRI guide to GIS analysis. Redlands: ESRI Press.

Pettit, C., \& Pullar, D. (1999). An integrated planning tool based upon miltiple criteria evaluation of spatial information. Computers, Environment and Urabn Systems, $23,339-357$.

Pick, J. (2005). Costs and Benefits of GIS in Business. In J. B. Pick, Geographic Information Systems in Business (pp. 56-78). Hershey: Idea Group Publishing.

Ritsema van Eck, J., \& de Jong, T. (1999). Accessibility analysis and spatial competition effects in the context of GIS-supported service location planning. Computers, Environment and Urabn Systems, 75-89.

Rogers, D. (2004, January 26). A status report on location analysis. Retrieved December 1, 2011, from DSN Retailing Today: http://findarticles.com/p/articles/mi_m0FNP/is_2_43/ai_112792451/

Schill, S. (2009, August ). Retrieved January 29, 2011, from SNV Netherlands Development Organisation: http://www.snvworld.org/en/Pages/default.aspx Todd, P., \& Benbasat, I. (2000). The impact of information technology on decision making: A cognitive perspective. In R. Zmud, Framing the Domain of IT Management (pp. 1-14). Cincinanati: Pinnaflex Education Resources. 


\section{Appendix A. Script for the Production Factors}

import arcpy

\#set the parameters

sectorGDB $=$ arcpy.GetParameterAsText $(0)$

sectorNumber $=$ arcpy.GetParameterAsText(1)

\#sum them all up

\#set the workspace

arcpy.env.workspace $=$ sectorGDB

table $=$ sectorGDB + "IISector" + str(sectorNumber)

\#check if the table exists

\# Select NAICS

NAICSNumber $=$ arcpy.GetParameterAsText $(2)$

naicslen $=$ len(NAICSNumber $)$

if naicslen $==5$ :

whereClause1 = "\"SUBbranch\" = " + str(NAICSNumber)

arcpy.MakeFeatureLayer_management(table, "sectorSelection", whereClause1) 
elif naicslen $==4$ :

whereClause2 = "।"Branchl" = l"' + str(NAICSNumber)

arcpy.MakeFeatureLayer_management(table, "sectorSelection", whereClause2)

elif naicslen $==3$ :

whereClause3 = "\"SubSector $\ "=l^{\prime \prime}+$ str(NAICSNumber)

arcpy.MakeFeatureLayer_management(table, "sectorSelection", whereClause3)

elif naicslen $==2$ :

whereClause4 = "I"Sector $"=$ I"' + str(NAICSNumber)

arcpy.MakeFeatureLayer_management(table, "sectorSelection", whereClause4)

else:

arcpy.AddError("Table doesn't exist")

\# set the parameters to get the weights from the user

\#set up a variable as integer SUM

remuneration_worker $=$ float $(\operatorname{arcpy} \cdot$ GetParameterAsText(3))

SUM = remuneration_worker

value_worker $=$ float $(\operatorname{arcpy}$. GetParameterAsText(4)) 
SUM = SUM + value_worker

grossma_worker $=$ float $(\operatorname{arcpy}$. GetParameterAsText(5))

SUM = SUM + grossma_worker

share_value $=$ float $(\operatorname{arcpy}$. GetParameterAsText(6))

SUM = SUM + share_value

imputed_capital $=$ float $(\operatorname{arcpy}$. GetParameterAsText(7))

SUM = SUM + imputed_capital

capital_ratio $=$ float $(\operatorname{arcpy}$. GetParameterAsText $(8))$

SUM = SUM + capital_ratio

total_firm = float $(\operatorname{arcpy}$. GetParameterAsText(9))

SUM = SUM + total_firm

average_firm = float(arcpy.GetParameterAsText(10))

SUM = SUM + average_firm

labor_intensity $=$ float $(\operatorname{arcpy}$. GetParameterAsText(11))

SUM = SUM + labor_intensity

VA_tproduction = float $(\operatorname{arcpy}$. GetParameterAsText $(12))$

SUM = SUM + VA_tproduction

revenue_worker $=$ float $(\operatorname{arcpy} \cdot$ GetParameterAsText(13))

$\mathrm{SUM}=\mathrm{SUM}+$ revenue_worker

size_industry = float(arcpy.GetParameterAsText(14))

SUM = SUM + size_industry

asset_turnover $=$ float $(\operatorname{arcpy}$. GetParameterAsText $(15))$

SUM = SUM + asset_turnover 
total_workers = float $(\operatorname{arcpy}$. GetParameterAsText(16))

$\mathrm{SUM}=$ SUM + total_workers

if not arcpy.Exists(table):

arcpy.AddError("Table for sector " + str(sectorNumber) + " doesn't exist")

else:

arcpy.AddMessage("DEBUG: " + table)

\#Calculate all the variables with the weight

arcpy.AddField_management("sectorSelection", "STD_Remuneration",

\section{"DOUBLE")}

\#the 100 change to sum of the weights

calcExpression $=("(!$ Rel_Remuneration! * " + str(remuneration_worker $)+$ " $) / "+$ $\operatorname{str}(\mathrm{SUM})+" ")$

arcpy.CalculateField_management("sectorSelection", "STD_Remuneration", calcExpression, "PYTHON")

arcpy.AddField_management("sectorSelection", "STD_Value", "DOUBLE")

calcExpression $1=("("+\operatorname{str}($ value_worker $)+$ " * !Rel_Value! $) /$ "+ str(SUM)+ "")

arcpy.CalculateField_management("sectorSelection","STD_Value",calcExpression1, "PYTHON") 
arcpy.AddField_management("sectorSelection", "STD_Grossma", "DOUBLE") calcExpression $2=("("+\operatorname{str}($ grossma_worker $)+$ "* !Rel_Grossma!) / "+ $\operatorname{str}(\mathrm{SUM})+" ")$

arcpy.CalculateField_management("sectorSelection","STD_Grossma",calcExpression2, "PYTHON")

arcpy.AddField_management("sectorSelection", "STD_Share", "DOUBLE") calcExpression3 = ("(" + str(share_value $)+" *$ !Rel_Share! $) /$ "+ str(SUM)+ "")

arcpy.CalculateField_management("sectorSelection","STD_Share",calcExpression3,"PY THON")

arcpy.AddField_management("sectorSelection", "STD_Imputed", "DOUBLE") calcExpression $4=("("+\operatorname{str}($ imputed_capital $)+$ * $*$ !Rel_Imputed! $) / "+\operatorname{str}($ SUM $)+$ "')

arcpy.CalculateField_management("sectorSelection","STD_Imputed",calcExpression4, "PYTHON")

arcpy.AddField_management("sectorSelection", "STD_Capital", "DOUBLE") calcExpression5 = ("(" + str(capital_ratio $)+$ " * !Rel_Capital! $) / "+\operatorname{str}(\mathrm{SUM})+$ " ") 
arcpy.CalculateField_management("sectorSelection","STD_Capital",calcExpression5, "PYTHON")

arcpy.AddField_management("sectorSelection", "STD_TFirms", "DOUBLE") calcExpression6 $=("("+\operatorname{str}($ total_firm $)+" *$ !Rel_TFirms! $) / \quad+\operatorname{str}(\mathrm{SUM})+$ "")

arcpy.CalculateField_management("sectorSelection","STD_TFirms",calcExpression6, "PYTHON")

arcpy.AddField_management("sectorSelection", "STD_AverageF", "DOUBLE") calcExpression7 = $("("+\operatorname{str}($ average_firm $)+" *$ !Rel_AverageF! $) / "+\operatorname{str}(\mathrm{SUM})+$ "')

arcpy.CalculateField_management("sectorSelection","STD_AverageF",calcExpression7, "PYTHON")

arcpy.AddField_management("sectorSelection", "STD_Labor", "DOUBLE") calcExpression8 = ("("+str(labor_intensity) + " * !Rel_Labor!) / "+ str(SUM)+ "")

arcpy.CalculateField_management("sectorSelection","STD_Labor",calcExpression8, "PYTHON") 
arcpy.AddField_management("sectorSelection", "STD_VA", "DOUBLE")

calcExpression9 = ("(" + str(VA_tproduction $)+" *$ !Rel_VA! $) / \quad+\operatorname{str}(\mathrm{SUM})+$ " $)$

arcpy.CalculateField_management("sectorSelection","STD_VA",calcExpression9, "PYTHON")

arcpy.AddField_management("sectorSelection","STD_Revenue", "DOUBLE")

calcExpression $10=("("+\operatorname{str}($ revenue_worker $)+" *$ Rel_Revenue! $) /$ "+ $\operatorname{str}(\mathrm{SUM})+" ")$

arcpy.CalculateField_management("sectorSelection","STD_Revenue",calcExpression10, "PYTHON")

arcpy.AddField_management("sectorSelection", "STD_SizeInd", "DOUBLE") calcExpression $11=("("+\operatorname{str}($ size_industry $)+" *$ !Rel_SizeInd!) $/$ "+ str(SUM)+

"")

arcpy.CalculateField_management("sectorSelection",

"STD_SizeInd",calcExpression11, "PYTHON")

arcpy.AddField_management("sectorSelection", "STD_AssetT", "DOUBLE")

calcExpression $12=("("+\operatorname{str}($ asset_turnover $)+" *$ !Rel_AssetT! $) /$ " + str(SUM)+

"") 
arcpy.CalculateField_management("sectorSelection","STD_AssetT",calcExpression12, "PYTHON")

arcpy.AddField_management("sectorSelection", "STD_TWorkers", "DOUBLE")

calcExpression13 = ("(" + str(total_workers $)+$ * !Rel_totworkers!) /"+

$\operatorname{str}(\mathrm{SUM})+" ")$

arcpy.CalculateField_management("sectorSelection","STD_TWorkers",calcExpression13 , "PYTHON")

\footnotetext{
arcpy.AddField_management("sectorSelection", "PF_Index", "DOUBLE") calcExpression14 = "!STD_Remuneration! + !STD_Value! + !STD_Grossma! + !STD_Share! + !STD_Imputed! + !STD_Capital! + !STD_TFirms! + !STD_AverageF! + !STD_Labor! + !STD_VA! + !STD_Revenue! + !STD_SizeInd! + !STD_AssetT! + !STD_TWorkers!"
}

arcpy.CalculateField_management("sectorSelection","PF_Index",calcExpression14, "PYTHON")

arcpy.Select_analysis("sectorSelection","Sector")

Sector $=$ sectorGDB + "IISector" 
\#display on the map

arcpy.MakeFeatureLayer_management(Sector,"ProductionFactors")

arcpy.SetParameterAsText(17,"ProductionFactors")

\section{Appendix B. Script for the Local Access}

import arcpy

\#set the parameters

sectorGDB $=$ arcpy.GetParameterAsText $(0)$

\#Transportation

company_location $=$ arcpy.GetParameterAsText $(1)$

company_state $=\operatorname{arcpy}$. GetParameterAsText $(2)$

highway_miles = float $(\operatorname{arcpy} . G e t P a r a m e t e r A s T e x t(3))$

port_miles $=$ float $(\operatorname{arcpy}$. GetParameterAsText(4))

rail_miles $=$ float $(\operatorname{arcpy}$. GetParameterAsText(5))

airport_miles $=$ float $(\operatorname{arcpy}$. GetParameterAsText(6))

highway_weight $=$ float $(\operatorname{arcpy}$. GetParameterAsText(7))

port_weight $=$ float $(\operatorname{arcpy}$. GetParameterAsText $(8))$

rail_weight $=$ float $(\operatorname{arcpy}$. GetParameterAsText(9))

airport_weight $=$ float $(\operatorname{arcpy} \cdot$ GetParameterAsText(10) $)$ 
\#set the workspace

arcpy.env.workspace $=$ sectorGDB

municentroid_miles $=$ sectorGDB + "IImunicentroid_miles"

\# preguntar por cero

arcpy.AddField_management("municentroid_miles", "Final_highwaymiles", "DOUBLE")

calcExpression15 =(" (" + str(highway_weight $)+$ " *(1-(!Near_HW!) $/$ "+ str(highway_miles)+"))")

arcpy.CalculateField_management("municentroid_miles", "Final_highwaymiles",calcExpression15, "PYTHON")

arcpy.AddField_management("municentroid_miles", "Final_portmiles", "DOUBLE") calcExpression16 =("(" + str(port_weight $)+$ " *(1-(!Near_Ports! $) / "+\operatorname{str}($ port_miles $)+$ "))")

arcpy.CalculateField_management("municentroid_miles", "Final_portmiles",calcExpression16, "PYTHON") 
arcpy.AddField_management("municentroid_miles", "Final_railmiles", "DOUBLE") calcExpression $17=("("+\operatorname{str}($ rail_weight $)+$ " *(1-(!Near_Rail! $) / "+\operatorname{str}($ rail_miles $)+$ "))")

arcpy.CalculateField_management("municentroid_miles", "Final_railmiles",calcExpression17, "PYTHON")

arcpy.AddField_management("municentroid_miles", "Final_airportmiles", "DOUBLE") calcExpression $18=("("+\operatorname{str}($ airport_weight $)+$ * $*(1-($ !Near_Airports! $) /$ " + str(airport_miles) + "))") arcpy.CalculateField_management("municentroid_miles", "Final_airportmiles",calcExpression18, "PYTHON")

arcpy.AddField_management("municentroid_miles", "Final_transportation", "DOUBLE")

calcExpression19 =("(!Final_highwaymiles! + !Final_portmiles! + !Final_railmiles! + !Final_airportmiles!)")

arcpy.CalculateField_management("municentroid_miles", "Final_transportation",calcExpression19, "PYTHON")

\#Company location 
cities_dtl = sectorGDB + "Ilcities_dtl"

\#

where_clause = "I"NAME\" =|" + str(company_location $)+" \mid '$ AND \"ST_ABBREV\" = I'" + str(company_state) + "'।"'

arcpy.Select_analysis("cities_dtl", "location", where_clause)

arcpy.Merge_management(["location", "municentroid_miles"], sectorGDB +

"Ilusamex_cities")

arcpy.AddField_management("usamex_cities", "total_distance", "DOUBLE")

arcpy.CalculateField_management("usamex_cities","total_distance","0")

calcExpression20 =("(!Dista_Border! + !Dist_Border!)")

arcpy.CalculateField_management("usamex_cities", "total_distance",calcExpression20, "PYTHON")

\#arcpy.SetParameterAsText(11,"usamex_cities")

minicipalities = sectorGDB + "IIminicipalities"

LocationAccess = sectorGDB + "\\LocationAccess"

arcpy.CopyFeatures_management(minicipalities,LocationAccess)

arcpy.JoinField_management("LocationAccess","MUN_NAME","usamex_cities","MUN _NAME") 
arcpy.Select_analysis("LocationAccess", "LocalAccess")

\#arcpy.RemoveJoin_management("municipality","usamex_cities")

arcpy.SetParameterAsText(11,"LocalAccess") 


\section{Appendix C. Script for the Final Results}

import arcpy

import math

\#set the parameters

sectorGDB $=$ arcpy.GetParameterAsText $(0)$

importance_distance $=$ float $(\operatorname{arcpy} . G e t P a r a m e t e r A s T e x t(1))$

importance_transportation $=$ float $(\operatorname{arcpy}$. GetParameterAsText $(2))$

importance_production $=$ float $(\operatorname{arcpy}$. GetParameterAsText(3))

\#set the workspace

arcpy.env. workspace $=$ sectorGDB

\#cities = sectorGDB = "Ilcities"

ProductionFactors $=$ "ProductionFactors"

LocalAccess = "LocalAccess"

\#arcpy.AddIndex_management("LocalAccess", "MUN_NAME","MUYY_INDEX")

\#arcpy.AddIndex_management("ProductionFactors", "Municipality", "Muy_INDEX")

arcpy.AddField_management("LocalAccess", "Final_pointscore", "DOUBLE")

arcpy.AddJoin_management(LocalAccess,"MUN_NAME",ProductionFactors,"Municipa lity", "KEEP_COMMON") 
\#arcpy.Select_analysis("LocalAccess", "BestLocations")

\#BestLocations = sectorGDB + "||BestLocations"

arcpy.CalculateField_management("LocalAccess","LocalAccess.Final_pointscore","0")

calcExpression $1=("((("+\operatorname{str}($ importance_transportation $)+" *$

!LocalAccess.Final_transportation! + " + str(importance_production) + " *

!Sector.Value_Municipality!)/100)/(((!LocalAccess.total_distance!/833.72)*100)*("

+str(importance_distance) $+" / 50))) "$ )

arcpy.CalculateField_management("LocalAccess",

"LocalAccess.Final_pointscore",calcExpression1, "PYTHON")

arcpy.Select_analysis("LocalAccess","BestLocations")

arcpy.SetParameterAsText(4,"BestLocations")

\#arcpy.MakeFeatureLayer_management(finalOutput,"Best Location")

arcpy.RemoveJoin_management("LocalAccess","Sector") 


\section{Appendix D. ModelBuilder Geodesic calculation}

Model Created by Drew Flater.

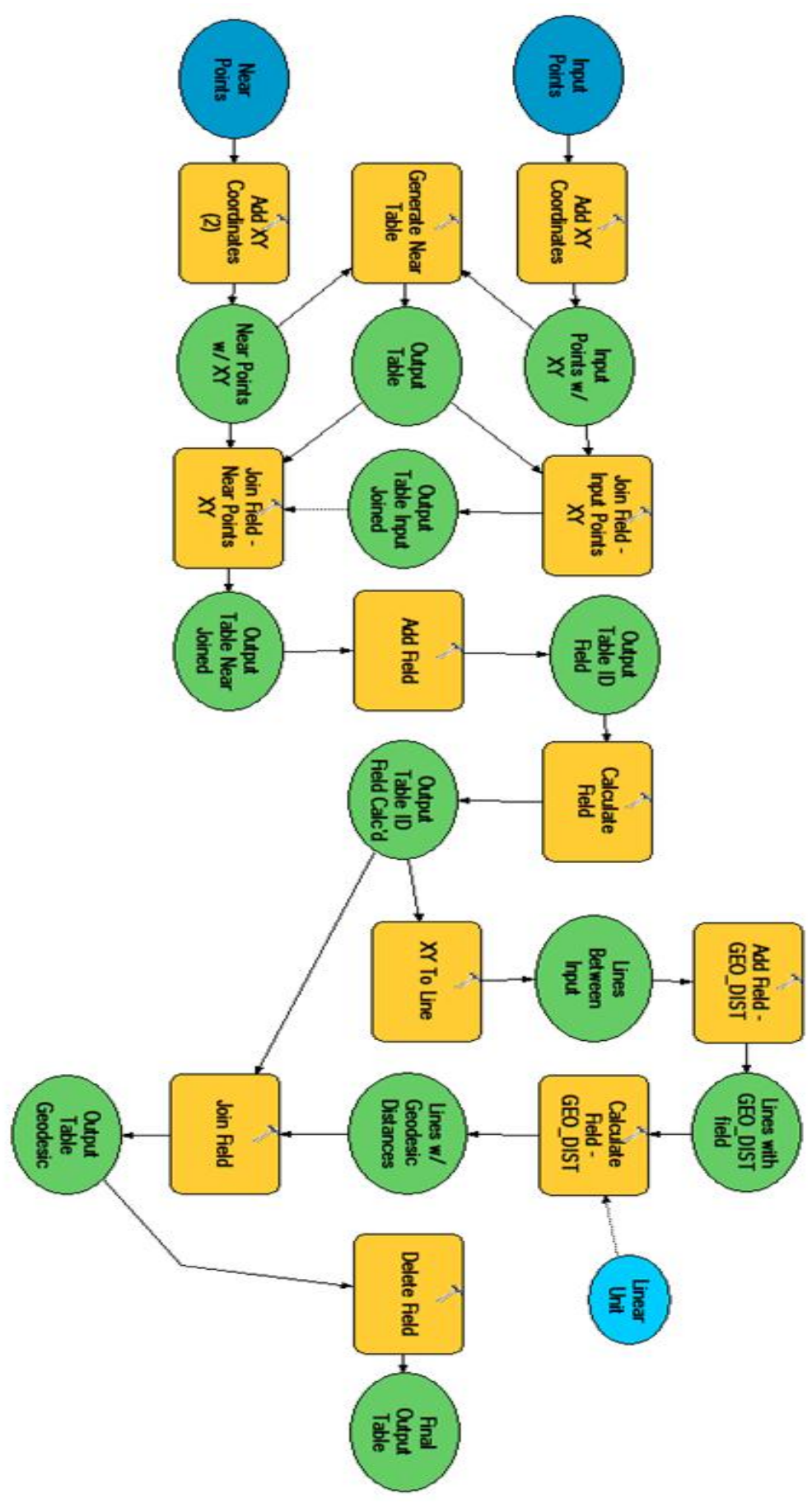

